\title{
Quark spins and anomalous Ward identity
}

\author{
Jian Liang, ${ }^{1, *}$ Yi-Bo Yang, ${ }^{2, \dagger}$ Terrence Draper, ${ }^{1}$ Ming Gong, ${ }^{3}$ and Keh-Fei Liu ${ }^{1, *}$
}

$(\chi \mathrm{QCD}$ Collaboration)

\author{
${ }^{1}$ Department of Physics and Astronomy, University of Kentucky, Lexington, Kentucky 40506, USA \\ ${ }^{2}$ Department of Physics and Astronomy, Michigan State University, East Lansing, Michigan 48824, USA \\ ${ }^{3}$ Institute of High Energy Physics, Chinese Academy of Sciences, Beijing 100049, China
}

(Received 29 June 2018; published 9 October 2018)

\begin{abstract}
We calculate the intrinsic quark spin contribution to the total proton spin using overlap valence quarks on three ensembles of 2+ 1-flavor RBC/UKQCD domain-wall configurations with different lattice spacings. The lowest pion mass of the ensembles is around $171 \mathrm{MeV}$, which is close to the physical point. With overlap fermions and a topological charge derived from the overlap operator, we verify the anomalous Ward identity between nucleon states with momentum transfer. Both the connected and the disconnected insertions of the axial-vector current are calculated. For the disconnected-insertion part, the clusterdecomposition error reduction technique is utilized for the lattice with the largest volume and the error can be reduced by $10 \%-40 \%$. Nonperturbative renormalization is carried out and the final results are all reported in the $\overline{\mathrm{MS}}$ scheme at $2 \mathrm{GeV}$. We determine the total quark spin contribution to the nucleon spin to be $\Delta \Sigma=0.405(25)(37)$, which is consistent with the recent global fitting result of experimental data. The isovector axial coupling we obtain in this study is $g_{A}^{3}=1.254(16)(30)$, which agrees well with the experimental value of $1.2723(23)$.
\end{abstract}

DOI: 10.1103/PhysRevD.98.074505

\section{INTRODUCTION}

The decomposition of the proton spin into its quark and glue constituents has long been a puzzle ever since the first deep inelastic scattering (DIS) experiment around three decades ago [1,2] revealed that not all the proton spin originates from the quark intrinsic spin as depicted in the naive quark model, leading to the so-called "proton spin crisis." Now we understand that the proton spin, consisting of quark spin, quark orbital angular momentum, glue spin, and glue orbital angular momentum, is the result of complicated QCD dynamics that cannot be described by the quark model. However, the precise proportion of the total proton spin carried by these components remains unclear. On the experimental side, since the integration of the spin-dependent parton distributions over the momentum fraction $x$ gives the fraction of the proton spin that is carried by the corresponding flavor, that is,

\footnotetext{
*jian.liang@uky.edu

yangyibo@pa.msu.edu

*liu@g.uky.edu
}

Published by the American Physical Society under the terms of the Creative Commons Attribution 4.0 International license. Further distribution of this work must maintain attribution to the author(s) and the published article's title, journal citation, and DOI. Funded by SCOAP ${ }^{3}$.

$$
\Delta q\left(\mu^{2}\right)=\int_{0}^{1} d x \Delta q\left(x, \mu^{2}\right),
$$

where $\mu$ is the $\overline{\mathrm{MS}}$ scale, the global fit to the experimental data of DIS or Drell-Yan processes for extracting the parton distributions will provide us knowledge about the quark spin contribution to the proton spin. Three recent experimental results from D. de Florian et al. [3], the NNPDF Collaboration [4], and the COMPASS Collaboration [5] determine the total quark intrinsic spin contribution $\Delta \Sigma$ to be $0.366_{-0.062}^{+0.042}, 0.25(10)$, and $[0.26,0.36]$, respectively. On the lattice side, a recent calculation [6] is carried out with the physical pion mass, but with only one single lattice ensemble of $N_{f}=2$ clover-improved twisted mass fermions. More careful lattice studies with ensembles of different lattice spacings and different lattice volumes are imperative to push the results to the physical limit and to control the corresponding systematic uncertainties.

In this work, we use overlap fermions on three domainwall ensembles to calculate the quark spin contribution to the nucleon spin. Since for each quark flavor the intrinsic spin is actually half of the corresponding axial coupling of the nucleon, we need to calculate the axial coupling for the flavor-diagonal case. Thus, both the connected insertions and the disconnected insertions of the correlation functions need to be included. The anomalous Ward identity is 
carefully checked to see if any normalization due to lattice artifacts needs to be applied to the axial-vector current to make the identity hold. We actually find that the same normalization constant for the local axial-vector current as used in the isovector case to satisfy the chiral Ward identity also satisfies the anomalous Ward identity. This is not true in general for nonchiral fermions. For the disconnectedinsertion part, the cluster-decomposition error reduction (CDER) technique [7] is utilized for the lattice with the largest volume to reduce the statistical error. For the connected-insertion part, an improved axial-vector current is employed such that the finite lattice spacing effect can be reduced. All of our results are matched to the $\overline{\mathrm{MS}}$ scheme at $2 \mathrm{GeV}$ using nonperturbative renormalization. We propose a new renormalization pattern where we separate the connected-insertion part and the disconnected-insertion part from the beginning, which is more natural for the lattice calculation and offers more information than the conventional flavor irreducible representation approach.

This paper is organized as follows. The formalism of quark spin and anomalous Ward identity are discussed in Sec. II. In Sec. III we describe all the numerical details of our simulation. Then in Sec. IV, we check the axial Ward identity to address the normalization issue. The bare results of the disconnected contribution are shown in Sec. V. The detailed results of the connected contribution come in Sec. VI. We discuss the renormalization in Sec. VII and make global fits to get the final results in Sec. VIII. A short summary is given in Sec. IX.

\section{FORMALISM OF QUARK SPIN AND ANOMALOUS WARD IDENTITY}

The quark spin contribution to the nucleon spin is associated with the nucleon matrix element of the flavorsinglet axial-vector current,

$$
g_{A}^{0} s_{\mu}=\frac{\left\langle p, s\left|A_{\mu}^{0}\right| p, s\right\rangle}{\langle p, s \mid p, s\rangle}
$$

where $A_{\mu}^{0}$ is the flavor-singlet axial-vector current

$$
A_{\mu}^{0}=\bar{\psi}_{u} i \gamma_{\mu} \gamma_{5} \psi_{u}+\bar{\psi}_{d} i \gamma_{\mu} \gamma_{5} \psi_{d}+\bar{\psi}_{s} i \gamma_{\mu} \gamma_{5} \psi_{s} .
$$

The flavor $u, d$, and $s$ contributions to $g_{A}^{0}$ are denoted as $\Delta u$, $\Delta d$, and $\Delta s$ in Eq. (2), so that

$$
g_{A}^{0}=\Delta u+\Delta d+\Delta s .
$$

A special property of the flavor-singlet axial-vector current is that it satisfies the anomalous Ward identity (AWI) where the Adler-Bell-Jackiw anomaly appears from the Jacobian factor of the fermion determinant due to the $U(1)$ chiral transformation [8]

$$
\partial_{\mu} A_{\mu}^{0}=\sum_{f=u, d, s} 2 m_{f} P_{f}-2 i N_{f} q
$$

where the pseudoscalar density $P_{f}$ and the topological charge density operator $q$ representing the anomaly are

$$
P_{f}=\bar{\psi}_{f} i \gamma_{5} \psi_{f}, \quad q=\frac{1}{16 \pi^{2}} G_{\mu \nu}^{a} \tilde{G}_{\mu \nu}^{a},
$$

where $G_{\mu \nu}^{a}$ is the gauge field strength tensor and $\tilde{G}_{\mu \nu}^{a}=\epsilon_{\mu \nu \rho \sigma} G_{\rho \sigma}^{a}$. Note that notations are in Euclidean space and the coupling constant $g$ is absorbed in the definition of the gauge potential $A_{\mu}^{a}$.

As far as renormalization is concerned, it is shown that $A_{\mu}^{0}$ has a two-loop renormalization $[9,10]$ and the topological charge has a one-loop mixture with $\partial_{\mu} A_{\mu}^{0}$ [10] so that the renormalized AWI in the dimensional regularization scheme becomes

$$
\begin{aligned}
\partial_{\mu} A_{\mu}^{0}\left(1+\gamma N_{f} \frac{1}{\epsilon}\right)= & \sum_{f=u, d, s} 2 m_{f}^{R} P_{f}^{R} \\
& +\left(-2 i N_{f} q+\gamma N_{f} \frac{1}{\epsilon} \partial_{\mu} A_{\mu}^{0}\right)
\end{aligned}
$$

with the anomalous dimension $\gamma=-\left(\alpha_{s} / \pi\right)^{2} \frac{3}{8} C_{F} \cdot m^{R}$ and $P^{R}$ are renormalized quark mass and pseudoscalar density. We see that the $\alpha_{s}^{2}$ renormalization term on the left is the same as that on the right from mixing. Thus, $m P$ and $\partial_{\mu} A_{\mu}^{0}+2 i N_{f} q$ are renormalization group invariant (the latter to second order at least), and the form of AWI is the same with or without renormalization.

On the lattice, the AWI is preserved by the overlap fermion that is chiral and satisfies the Ginsparg-Wilson relation [11]. The $m_{f} P_{f}$ is renormalization group invariant since $Z_{m} Z_{P}=1$ for the chiral fermion and the local version of the topological charge $q(x)$ derived from the overlap operator is equal to $\frac{1}{16 \pi^{2}} \operatorname{tr}_{c} G_{\mu \nu} \tilde{G}_{\mu \nu}(x)$ in the continuum [12-15], i.e.,

$$
q(x)=\operatorname{Tr}\left[\gamma_{5}\left(\frac{1}{2} D_{\mathrm{ov}}(x, x)-1\right)\right] \underset{a \rightarrow 0}{\longrightarrow} \frac{1}{16 \pi^{2}} \operatorname{tr}_{c} G_{\mu \nu} \tilde{G}_{\mu \nu}(x),
$$

where $D_{\text {ov }}$ is the overlap operator. In the overlap case, the chiral axial-vector current is derived [16], and one can directly proceed to carry out the renormalization of the chiral axial-vector current perturbatively or nonperturbatively. However, this chiral axial vector involves a nonlocal kernel $K_{\mu}=-\left.i \frac{\delta D_{\mathrm{ov}}\left(U_{\mu} e^{i \alpha_{\mu}(x)}\right)}{\delta \alpha_{\mu}(x)}\right|_{\alpha=0}$ and is somewhat involved to implement numerically. We shall use the local current in the present study. As such, it invokes a normalization constant $Z_{A}^{0}$ which warrants that the unrenormalized AWI 
in its "semiclassical" form [Eq. (5)] is satisfied on the lattice and is itself scale independent. Therefore the normalization and renormalization takes two steps. First, one needs to find the normalization $Z_{A}^{0}$ for the local axial-vector current that satisfies the unrenormalized AWI

$$
\partial_{\mu} Z_{A}^{0} A_{\mu}^{0}=\sum_{f=u, d, s} 2 m_{f} P_{f}-2 i N_{f} q
$$

where $A_{\mu}^{0}=\sum_{f=u, d, s} \bar{\psi}_{f} i \gamma_{\mu} \gamma_{5} \hat{\psi}_{f}$ and $P_{f}=\bar{\psi}_{f} i \gamma_{5} \hat{\psi}_{f}$ are the local axial-vector current we use on the lattice and $\hat{\psi}=$ $\left(1-\frac{1}{2} D_{\mathrm{ov}}\right) \psi$ is for giving rise to the effective quark propagator that conforms to the form in the continuum.

After the normalization constant $Z_{A}^{0}$ is determined, one then takes on the renormalization procedure. We shall discuss the determination of $Z_{A}^{0}$ in Sec. IV after we give the numerical details of the calculation, and we will carry out the renormalization in Sec. VII.

Before we check the AWI on the lattice, we shall first give some numerical details of the lattice calculation.

\section{NUMERICAL DETAILS}

We use overlap fermions [17] as valence quarks to perform our calculation. Since the overlap action preserves chiral symmetry at finite lattice spacing via the GinspargWilson relation [18], there is no additive renormalization for the quark mass. The effective quark propagator of the massive overlap fermion is the inverse of operator $D_{c}+m$ $[19,20]$ where $D_{c}$ satisfying $\left\{D_{c}, \gamma_{5}\right\}=0$ is exactly chiral and can be defined from the original overlap operator $D_{\mathrm{ov}}$ as $D_{c}=\frac{\rho D_{\mathrm{ov}}}{1-D_{\mathrm{ov}} / 2}$. The overlap operator can be expressed as $D_{\mathrm{ov}}=1+\gamma_{5} \epsilon\left(\gamma_{5} D_{\mathrm{w}}(\rho)\right)$ where $\epsilon$ is the matrix sign function and $D_{\mathrm{w}}$ is the Wilson kernel with $\kappa=0.2$ (corresponding to parameter $\rho=1.5$ ). As discussed above, another great feature of the overlap operator is that the local version of the topological charge of the gauge field can be defined as $q(x)=\operatorname{Tr}\left[\gamma_{5}\left(\frac{1}{2 \rho} D_{\text {ov }}(x, x)-1\right)\right]$ [12-15]. The AtiyahSinger index theorem [21] is satisfied, which relates the total topological charge to the index of zero modes of the overlap operator so no multiplicative renormalization is needed for this definition of $q$. These two features help us to feasibly check the AWI, which we can use as a normalization condition in the disconnected-insertion case. We use multiple partially quenched valence quark masses to cover a wide range of pion mass using the multimass algorithm. More details regarding the calculation of the overlap operator and eigenmode deflation in the inversion of the fermion matrix can be found in [22].

The three lattice ensembles we use for the calculation are $2+1$-flavor domain-wall fermion (DWF) ensembles generated by the RBC/UKQCD Collaboration [23,24]. They are labeled as 24I, 32I, and 32ID, and the detailed parameters of the ensembles can be found in Table I.
TABLE I. The parameters of the $2+1$-flavor RBC/UKQCD configurations: label, spatial/temporal size, lattice spacing, the degenerate light sea quark mass, strange sea quark mass, the corresponding pion mass, and the number of configurations used in this work.

\begin{tabular}{lcccccc}
\hline \hline Label & $L^{3} \times T$ & $a^{-1}[\mathrm{GeV}]$ & $m_{l}^{(s)} a$ & $m_{s}^{(s)} a$ & $m_{\pi}[\mathrm{MeV}]$ & $N_{\text {cfg }}$ \\
\hline 32I & $32^{3} \times 64$ & $2.3833(86)$ & 0.004 & 0.03 & 302 & 309 \\
24I & $24^{3} \times 64$ & $1.7848(50)$ & 0.005 & 0.04 & 337 & 203 \\
32ID & $32^{3} \times 64$ & $1.3784(68)$ & 0.001 & 0.045 & 171 & 200 \\
\hline \hline
\end{tabular}

We have three different lattice spacings, and the lowest pion mass at $171 \mathrm{MeV}$ is close to the physical one.

To calculate the quark spin or, in practice, to calculate the axial coupling, we need to construct three-point correlation functions

$$
C_{3, \mu}\left(t_{f}, \tau\right)=\sum_{\vec{x}, \vec{y}}\left\langle\chi\left(t_{f}, \vec{y}\right) A_{\mu}(\tau, \vec{x}) \bar{\chi}(0, \mathcal{G})\right\rangle
$$

where $\chi$ is the nucleon interpolation field, $\mathcal{G}$ denotes the source grid, and $A_{\mu}=\bar{\psi} i \gamma_{\mu} \gamma_{5} \hat{\psi}$ is the local axial-vector current with $\hat{\psi}=\left(1-\frac{1}{2} D_{\mathrm{ov}}\right) \psi$ for giving rise to the effective quark propagator $\left(D_{c}+m\right)^{-1}$. The correlation function can have two kinds of current insertions, i.e., the connected insertion (CI) and the disconnected insertion (DI), corresponding to two ways of Wick contractions. They are depicted in Fig. 1.

For the CI calculation, we use the stochastic sandwich method (SSM) with low-mode substitution (LMS) [25] to better control the statistical uncertainty. We use $Z_{3}$ noise grid sources with Gaussian (24I and 32I) or block smearing (32ID) [26] coherently at $t_{\text {src }}=0$ and $t_{\text {src }}=32$ in one inversion. The sinks are block smeared and located at different positions with different separations in time from the source. Setups regarding the valence simulation of the $\mathrm{CI}$ case are listed in Table II. Technical details regarding the LMS of random $Z_{3}$ grid source with mixed momenta and the SSM with LMS for constructing three-point functions can be found in Refs. [25-27]. Because of the fact that the multimass inversion algorithm is uniquely applicable to the overlap fermion with eigenvector deflation, we calculate 5-6 valence masses each for the three lattices.
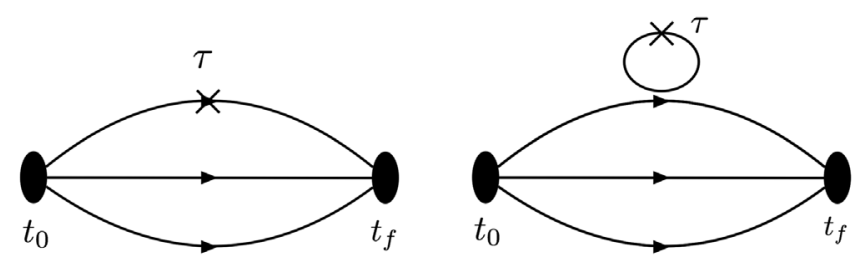

FIG. 1. The connected insertion (left) and disconnected insertion (right). 
TABLE II. The details of the overlap simulation in the valence sector for the CI case, including the name of the lattice, the grid type of source $\mathcal{G}_{\text {src }}$ (the notations such as 12-12-12 denote the intervals of the grid in the three spatial directions; see Ref. [25] for more details), the number of source grids $N_{\text {src }}$, the positions of sources $t_{\text {src }}$, the grid type of sink $\mathcal{G}_{\text {sink }}$, the number of the noises for the sink grids $N_{\text {sink }}$, the source-sink separations $\left(t_{\text {sink }}-t_{\text {src }}\right)$, and the bare valence quark masses $m_{q}^{v} a$.

\begin{tabular}{|c|c|c|c|c|c|c|c|}
\hline Lattice & $\mathcal{G}_{\mathrm{src}}$ & $N_{\text {src }}$ & $t_{\mathrm{src}}$ & $\mathcal{G}_{\text {sink }}$ & $N_{\text {sink }}$ & $\left(t_{\text {sink }}-t_{\text {src }}\right)$ & $m_{q}^{v} a$ \\
\hline \multirow[t]{4}{*}{$24 \mathrm{I}$} & $12-12-12$ & 1 & $(0,32)$ & $2-2-2$ & 5 & $0.88 \mathrm{fm}$ & $(0.00809,0.0102,0.0135,0.0160,0.0203)$ \\
\hline & & & & & 3 & $1.11 \mathrm{fm}$ & \\
\hline & & & & & 5 & $1.22 \mathrm{fm}$ & \\
\hline & & & & & 5 & $1.33 \mathrm{fm}$ & \\
\hline \multirow[t]{3}{*}{$32 I$} & $16-16-16$ & 1 & $(0,32)$ & $1-1-1$ & 3 & $0.99 \mathrm{fm}$ & $(0.00585,0.00765,0.00885,0.0112,0.0152)$ \\
\hline & & & & & 3 & $1.16 \mathrm{fm}$ & \\
\hline & & & & & 3 & $1.24 \mathrm{fm}$ & \\
\hline \multirow[t]{5}{*}{ 32ID } & $16-16-16$ & 6 & $(0,32)$ & $1-1-1$ & 2 & $1.00 \mathrm{fm}$ & $(0.0042,0.0060,0.011,0.014,0.017,0.022)$ \\
\hline & & & & & 3 & $1.15 \mathrm{fm}$ & \\
\hline & & & & & 4 & $1.29 \mathrm{fm}$ & \\
\hline & & & & & 5 & $1.43 \mathrm{fm}$ & \\
\hline & & & & & 12 & $1.57 \mathrm{fm}$ & \\
\hline
\end{tabular}

For disconnected-insertion calculations, we use the lowmode average (LMA) technique to calculate the quark loops, which improves the signal-to-noise ratio particularly for the pseudoscalar and scalar currents. The low-mode part is calculated exactly while the high-mode part is estimated with eight sets of $Z_{4}$-noise on a 4-4-4-2 spacetime grid with even-odd dilution and additional time shift. The same $Z_{3}$-noise grid source with smearing as in the CI case is used in the production of the nucleon propagators. We make multiple measurements by shifting the source time slice to improve statistics; the spatial position of the center of the grid is randomly chosen for each source time slice to reduce autocorrelation. References [27-29] contain more details regarding the DI calculation. When constructing quark loops, we include more valence quark masses to cover the strange region. The bare strange quark mass is determined by the global-fit value at $2 \mathrm{GeV}$ in the $\overline{\mathrm{MS}}$ scale calculated in our previous study [30] and the nonperturbative mass renormalization constant calculated in [31].

To obtain the axial coupling, we construct a ratio of the three-point correlation function to the nucleon two-point function

$$
R\left(t_{f}, \tau\right)=f_{k} \frac{\operatorname{Tr}\left[\Gamma_{p} C_{3}\left(t_{f}, \tau\right)\right]}{\operatorname{Tr}\left[\Gamma_{e} C_{2}\left(t_{f}\right)\right]}
$$

where $f_{k}$ is a kinematic factor that is related to the Lorentz index of the current, $\Gamma_{p}$ is the polarized projector of the nucleon spin, $\Gamma_{e}$ is the nonpolarized projector, and $C_{2}\left(t_{f}\right)=\sum_{\vec{x}}\left\langle\chi\left(t_{f}, \vec{x}\right) \bar{\chi}(0, \mathcal{G})\right\rangle$. The matrix element $g_{A}$ can then be obtained asymptotically $g_{A}=R\left(t_{f} \gg \tau, \tau \gg 0\right)$. However, at finite $t_{f}$ and $\tau$, the excited states will contribute to the ratio, and we need to extract $g_{A}$ by fitting the ratio to more complicated function forms. A commonly used form with a two-state fit reads

$$
R\left(t_{f}, \tau\right)=g_{A}+c_{1} e^{-\delta m\left(t_{f}-\tau\right)}+c_{2} e^{-\delta m \tau}+c_{3} e^{-\delta m t_{f}},
$$

which assumes only the first excited state has effects and $\delta m$ is the energy difference between the ground state and the first excited state. In practice, higher excited-states' contribution can alter the value of $\delta m$, making it a free parameter, accounting for an effective energy difference.

\section{ANOMALOUS WARD IDENTITY ON THE LATTICE}

To verify the AWI in Eq. (9), we note that there is no flavor mixing in this unrenormalized form. Thus, one can check it for individual flavors and, furthermore, since the lattice calculations of matrix elements are separated in the CI and DI cases as shown in Fig. 1, one can separately check the chiral Ward identity for the connected matrix elements

$$
\left\langle p^{\prime}\left|\partial_{\mu} Z_{A}(\mathrm{CI}) A_{\mu}\right| p\right\rangle(\mathrm{CI})=\left\langle p^{\prime}\left|2 m_{q} P\right| p\right\rangle(\mathrm{CI}) .
$$

In this case, the matrix elements are for the $u$ or $d$ valence quark with quark mass $m_{q}$. Here, the normalization constant $Z_{A}(\mathrm{CI})$ is due to the fact that we use the local current in this calculation.

Similarly, the AWI for the matrix elements in the DI case is

$\left\langle p^{\prime}\left|\partial_{\mu} Z_{A}(\mathrm{DI}) A_{\mu}\right| p\right\rangle(\mathrm{DI})=\left\langle p^{\prime}\left|2 m_{q} P-2 i q\right| p\right\rangle(\mathrm{DI})$.

In principle, the normalization constants $Z_{A}(\mathrm{CI})$ and $Z_{A}(\mathrm{DI})$ can be different, especially when nonchiral fermions are used in the lattice calculation and also when the topological charge $q$ is not calculated from the overlap operator $D_{\mathrm{ov}}$ as in Eq. (8). We shall check them in the following. 


\section{A. Disconnected insertion case}

We shall check first the DI case that has been investigated in our previous study [28]. The AWI in the DI case in Eq. (14) relating the nucleon matrix element of the divergence of the axial-vector current $A_{\mu}$ to those of the product of the quark mass $m_{q}$ and the pseudoscalar current $P$ and also to the topological charge term $q$ is an important check for lattice spin calculations involving the flavordiagonal matrix elements (MEs) of the axial-vector current. This is especially true for the strange quark as it only contributes in the DI. Only properly extracted MEs plus correct lattice normalization will make this identity hold. Our previous work [28] utilized the AWI via the form factors, which is actually an extended form of the Goldberger-Treiman relation for the flavor-singlet case at finite momentum transfer $q^{2}$ and is expressed as

$$
g_{A}\left(q^{2}\right)+\frac{q^{2}}{2 m_{N}} h_{A}\left(q^{2}\right)=\frac{2 m_{q}}{2 m_{N}} g_{P}\left(q^{2}\right)+2 g_{Q}\left(q^{2}\right),
$$

where $g_{A}$ and $h_{A}$ are the axial and induced pseudoscalar form factors, respectively, from the nucleon matrix element of the axial-vector current

$$
\left\langle p^{\prime}, s\left|A_{\mu}\right| p, s\right\rangle=\bar{u}\left(p^{\prime}, s\right)\left[i \gamma_{\mu} \gamma_{5} g_{A}\left(q^{2}\right)-i q_{\mu} \gamma_{5} h_{A}\left(q^{2}\right)\right] u(p, s),
$$

and $g_{P}\left(q^{2}\right)$ and $g_{Q}\left(q^{2}\right)$ are the pseudoscalar and anomaly form factors defined in

$$
\begin{array}{r}
\left\langle p^{\prime}, s|P| p, s\right\rangle=\bar{u}\left(p^{\prime}, s\right) i \gamma_{5} u(p, s) g_{P}\left(q^{2}\right), \\
\left\langle p^{\prime}, s|-i q| p, s\right\rangle=\bar{u}\left(p^{\prime}, s\right) i \gamma_{5} u(p, s) m_{N} g_{Q}\left(q^{2}\right) .
\end{array}
$$

Equations (16) and (17) have the same form separately for the CI and DI, while Eq. (18) for the topological form factor only appears in the DI. Equation (15) can be derived by inserting the currents between nucleon states with momenta $\vec{p}$ and $\overrightarrow{p^{\prime}}$ and applying the divergence to the nucleon states

$\partial_{\mu}\left\langle p^{\prime}\left|A_{\mu}\right| p\right\rangle=\left(E^{\prime}-E\right)\left\langle p^{\prime}\left|A_{4}\right| p\right\rangle+i q_{i}\left\langle p^{\prime}\left|A_{i}\right| p\right\rangle$,

where $E$ and $E^{\prime}$ are the energies of the source and sink nucleons and $q_{i}$ is the momentum transfer $\vec{q}=\overrightarrow{p^{\prime}}-\vec{p}$ in the $i$ th direction. In the earlier study [28] we found that a normalization factor of $\kappa_{A} \sim 1.4$ on the axial-vector side is needed in order to satisfy the identity that is much larger than the isovector normalization constant ${ }^{1} Z_{A}^{3}(24 \mathrm{I})=$ 1.111(6) computed by using the chiral Ward identity in the pion two-point function case [31]. Since on the righthand side of Eq. (15) we have $Z_{m} Z_{P}=1$, there is no multiplicative renormalization of the topological charge defined by the overlap operator, and, as shown previously, the renormalized ${ }^{2}$ AWI is the same as the unrenormalized one at the two-loop level [28], the factor $\kappa_{A}$ was believed to be a necessary normalization factor in the DI case for compensating the violation of the AWI induced by lattice artifacts and was used to normalize the DI axial-vector MEs. In this study, we shall have a critical reexamination of this issue. We also make a similar check for the light quarks case of 24I and 32I and for the new 32ID lattice by calculating the following ratio:

$$
R_{1}\left(\tau, t_{f}, q^{2}\right)=\frac{\frac{2 m_{q}}{2 m_{N}} g_{P}\left(\tau, t_{f}, q^{2}\right)+2 g_{Q}\left(\tau, t_{f}, q^{2}\right)}{g_{A}\left(\tau, t_{f}, q^{2}\right)+\frac{q^{2}}{2 m_{N}} h_{A}\left(\tau, t_{f}, q^{2}\right)} .
$$

Note that we keep the dependence of the sink time $t_{f}$ and the current time $\tau$ for all the form factors and, therefore, the excited-state effects are not handled until we fit the final ratio.

We also check the AWI more carefully at the ME level, in other words, treating $\partial_{\mu} A_{\mu}$ as an operator insertion between the nucleon states $p$ and $p^{\prime}$. The lattice version of the AWI reads

$$
\sum_{\vec{x}}\left(\sum_{i}\left\langle A_{i}(\tau, \vec{x})-A_{i}(\tau, \vec{x}-\hat{i})\right\rangle+\left\langle A_{4}(\tau, \vec{x})-A_{4}(\tau-1, \vec{x})\right\rangle\right) e^{-i \vec{q} \cdot \vec{x}}=\frac{2 m_{q}}{2 m_{N}}\langle P(\tau, \vec{q})\rangle-2 i\langle q(\tau, \vec{q})\rangle,
$$

where $\left\langle A_{i}(\tau, \vec{x})\right\rangle$ is an abbreviated form of the $\mathrm{ME}$ $\left\langle p^{\prime}\left|A_{i}(\tau, \vec{x})\right| p\right\rangle, \hat{i}$ is the unit vector along the $i$ th direction, and the continuum partial derivative is replaced by the

\footnotetext{
${ }^{1}$ We choose to call it a normalization constant rather than a renormalization constant since it is a finite renormalization that has no scale dependence and deviates from unity only because of the finite lattice spacing effects.

${ }^{2}$ When we say renormalization, we mean there is a nonzero anomalous dimension and therefore is scale dependent.
}

backward difference on the lattice. This equation cannot be checked directly since the momentum projection is always done before we can take the spatial difference $A_{i}(\tau, \vec{x})-$ $A_{i}(\tau, \vec{x}-\hat{i})$ in the three-point function calculation. However,

$$
\begin{aligned}
& \sum_{\vec{x}} \sum_{i}\left\langle A_{i}(\tau, \vec{x})-A_{i}(\tau, \vec{x}-\hat{i})\right\rangle e^{-i \vec{q} \cdot \vec{x}} \\
& \quad=\left(1-e^{-i q_{i}}\right) \sum_{\vec{x}}\left\langle A_{i}(\tau, \vec{x})\right\rangle e^{-i \vec{q} \cdot \vec{x}} \sim i q_{i}\left\langle A_{i}(\tau, \vec{q})\right\rangle
\end{aligned}
$$



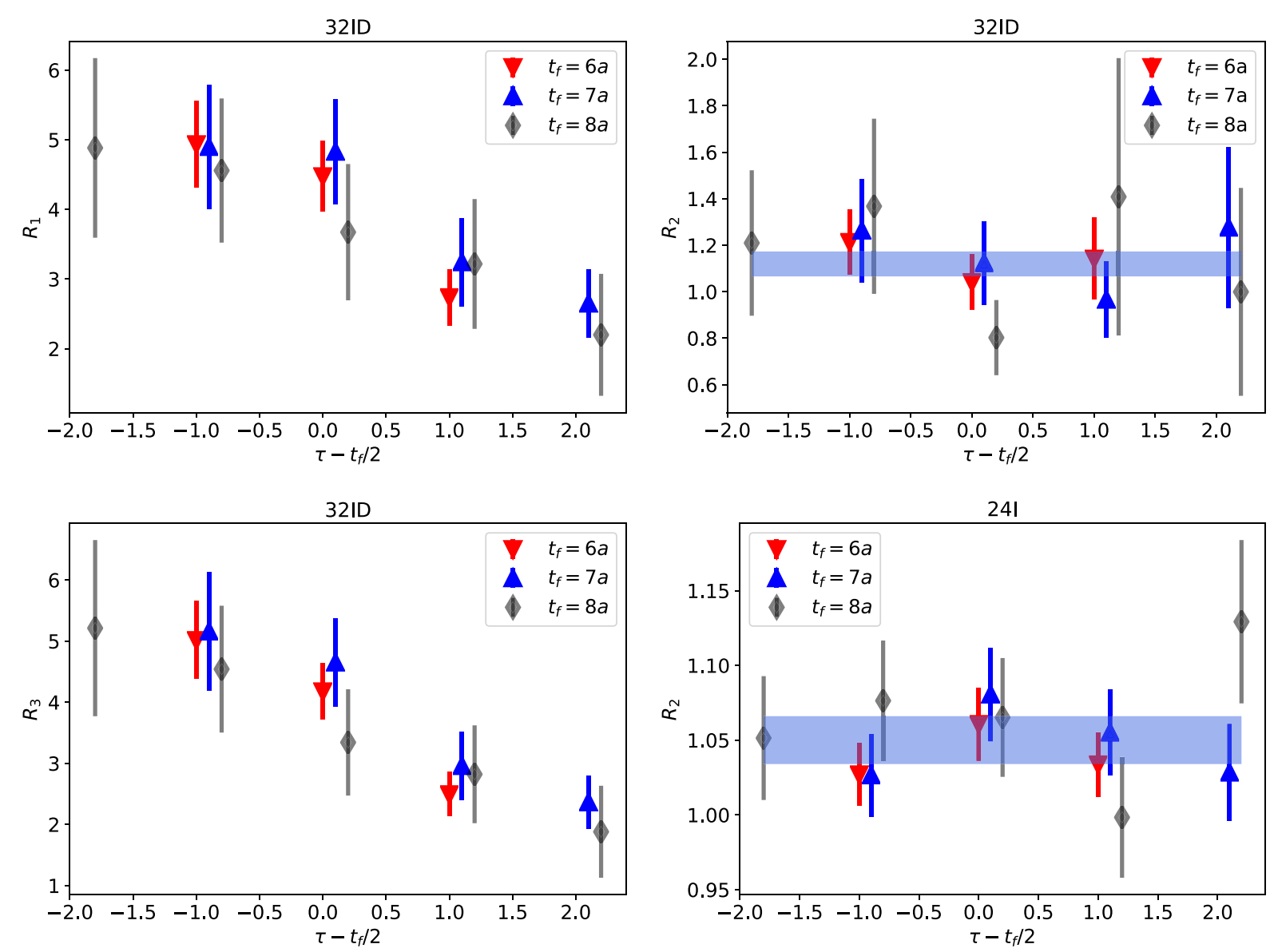

FIG. 2. Ratios $R_{1}, R_{2}$, and $R_{3}$ on the 32ID lattice and the ratio $R_{2}$ on the 24I lattice around the unitary points with momentum transfer $|\vec{q}|=\frac{2 \pi}{L}$ are plotted as a function of $\tau-\frac{t_{f}}{2}$. Three source-sink separations $t_{f}$ are included. The blue bands show the constant fit results of $R_{2}$. Points of different $t_{f}$ are shifted slightly to enhance the legibility, and the transparency of the points with $t_{f}=8 a$ is increased for the same purpose.

is a good approximation if $q_{i}$ is small enough. So we have a simplified form

$$
\begin{gathered}
i q_{i}\left\langle A_{i}(\tau, \vec{q})\right\rangle+\left\langle A_{4}(\tau, \vec{q})-A_{4}(\tau-1, \vec{q})\right\rangle \\
=\frac{2 m_{q}}{2 m_{N}}\langle P(\tau, \vec{q})\rangle-2 i\langle q(\tau, \vec{q})\rangle
\end{gathered}
$$

which can be checked numerically. A second ratio $R_{2}$ is thus defined as

$$
R_{2}=\frac{\frac{2 m_{q}}{2 m_{N}}\langle P(\tau, \vec{q})\rangle-2 i\langle q(\tau, \vec{q})\rangle}{i q_{i}\left\langle A_{i}(\tau, \vec{q})\right\rangle+\left\langle A_{4}(\tau, \vec{q})-A_{4}(\tau-1, \vec{q})\right\rangle}
$$

Furthermore, for the temporal part, by inserting complete sets of intermediate states and using the time evolution operator, the time dependence of the ME can be formulated as

$$
\left\langle A_{4}(\tau, \vec{x})\right\rangle=\left\langle A_{4}(0, \vec{q})\right\rangle e^{+\Delta E \tau}
$$

up to exponential suppression of the excited-states contamination, where $\Delta E=E^{\prime}-E$ is the energy difference between the sink and the source nucleon states, such that

$$
\left\langle A_{4}(\tau, \vec{x})-A_{4}(\tau-1, \vec{x})\right\rangle \sim \Delta E\left\langle A_{4}(\tau, \vec{x})\right\rangle,
$$

leading to

$i q_{i}\left\langle A_{i}(\tau, \vec{q})\right\rangle+\Delta E\left\langle A_{4}(\tau, \vec{q})\right\rangle=2 m_{q}\langle P(\tau, \vec{q})\rangle-2 i\langle q(\tau, \vec{q})\rangle$

if the excited-states contamination can be ignored or completely removed by fit. This is actually the counterpart of Eq. (19), and we now have the third ratio to check the AWI

$$
R_{3}=\frac{\frac{2 m_{q}}{2 m_{N}}\langle P(\tau, \vec{q})\rangle-2 i\langle q(\tau, \vec{q})\rangle}{i q_{i}\left\langle A_{i}(\tau, \vec{q})\right\rangle+\Delta E\left\langle A_{4}(\tau, \vec{q})\right\rangle} .
$$

The numerical results of ratios $R_{1}, R_{2}$, and $R_{3}$ on the 32ID lattice around the unitary point with momentum transfer $|\vec{q}|=\frac{2 \pi}{L}$ are plotted in Fig. 2 as a function of $t$. 

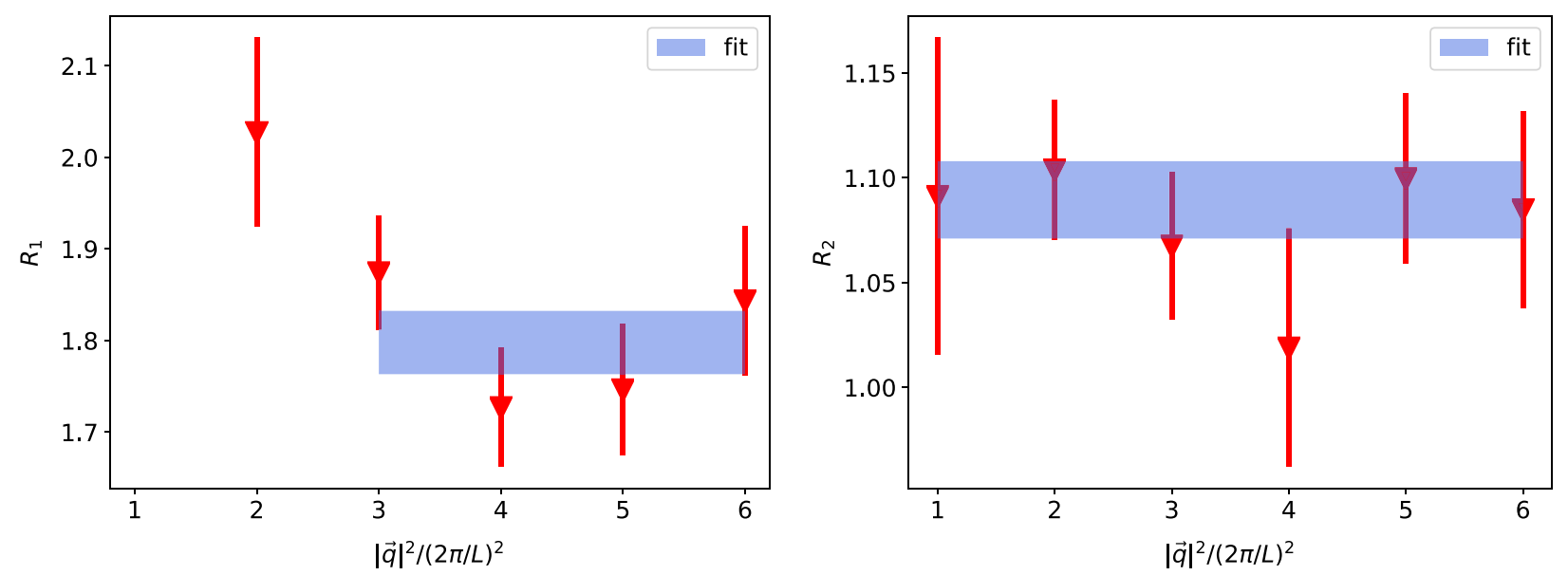

FIG. 3. The momentum transfer dependence of $R_{1}$ and $R_{2}$ on the 32ID lattice at the unitary point. The blue bands are the constant fit results. Each point comes from a fit combining different $t_{f}$. The point at the first momentum transfer in the left panel is missing because the corresponding two-state fit fails.

Three $t_{f}$ are included so the $t_{f}$ dependence can be checked. It can be seen that $R_{3}$ (lower left panel) is merely slightly different from $R_{1}$ (upper left panel) and they agree with each other quite well within errors, meaning that there would be no difference regardless of whether we check the AWI on the form factor level or on the ME level with the partial derivatives replaced by energy and momentum transfer in the latter case. This also serves as a sanity check of our calculation. The values of $R_{1}$ or $R_{3}$ are far away from 1 and are not flat versus $t$, making it very hard to have a reliable fit. But the situation of $R_{2}$ in the upper right panel is much different. The points are more regular and a value of 1.091(76) can be easily extracted by a constant fit, which is quite consistent with the isovector normalization constant $Z_{A}^{3}(32 \mathrm{ID})=1.141(1)$ computed using pion twopoint functions as in Ref. [31]. The ratio of $R_{2}$ on the 24I lattice is also calculated and shown in the lower right panel

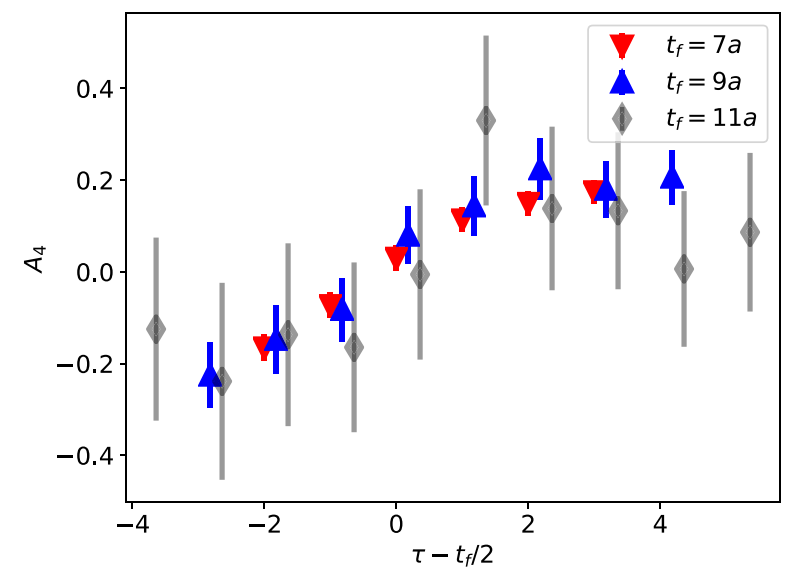

of Fig. 2. Unlike the normalization factor $k_{A} \sim 1.4$ obtained in the previous study through the ratio $R_{1}$ by using form factors, $R_{2}$ is also consistent with the isovector normalization constant $Z_{A}^{3}(24 \mathrm{I})=1.111(6)$ where the value from a constant fit is $1.074(24)$.

The momentum transfer dependences of $R_{1}$ and $R_{2}$ are plotted in Fig. 3. Each point on the plot of $R_{1}$ comes from a two-state fit while the points on the plot of $R_{2}$ come from constant fits. In the $R_{1}$ plot, except for the first two $|\vec{q}|^{2}$ (the point of the first $|\vec{q}|^{2}$ is not shown in the figure since the corresponding two-state fit fails), the values are basically flat within errors and the fitted value of a constant fit is 1.798(35). If we believe that the ratio $R_{1}$ is a proper check of the AWI, this value should be used as a normalization factor. In the $R_{2}$ case, all the points lie on a constant line within errors and a constant fit excluding the fourth point gives 1.096(15), quite consistent with $Z_{A}^{3}(24 \mathrm{I})=1.111(6)$.

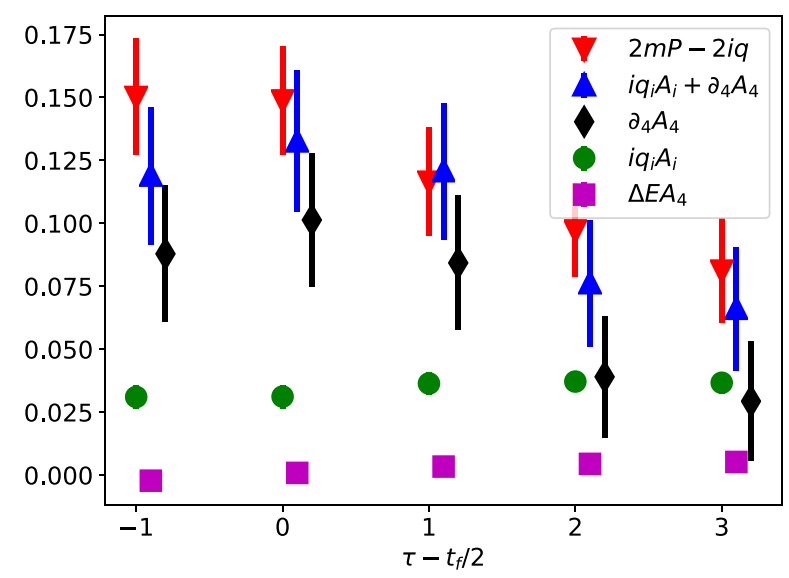

FIG. 4. The behavior of $\left\langle A_{4}\right\rangle$ with respect to $\tau$ and $t_{f}$ (left panel) and the components of the AWI at $t_{f}=7 a$ (right panel). The results are from the 32ID lattice at the unitary points with momentum transfer $|\vec{q}|=\frac{2 \pi}{L}$. The legend $\partial_{4} A_{4}$ stands for the $\left\langle A_{4}(\tau, \vec{q})-A_{4}(\tau-1, \vec{q})\right\rangle$ term. Points of different $t_{f}$ are shifted slightly to enhance the legibility, and the transparency of the points with $t_{f}=11 a$ in the left panel is increased for the same purpose. 
The problem now is to understand the discrepancy and to determine which one is correct.

Since $R_{1}$ and $R_{3}$ in Fig. 2 are quite similar, we shall only compare $R_{3}$ and $R_{2}$. It is easy to see that the only difference between $R_{2}$ and $R_{3}$ is to use $\left\langle A_{4}(\tau, \vec{q})-A_{4}(\tau-1, \vec{q})\right\rangle$ or $\Delta E\left\langle A_{4}(\tau, \vec{q})\right\rangle$. We have proven that they are exactly the same if there are no excited-state effects. So it is useful to see what $\left\langle A_{4}(\tau, \vec{q})\right\rangle$ looks like. The left panel of Fig. 4 shows the behavior of $\left\langle A_{4}\left(\tau,|\vec{q}|=\frac{2 \pi}{L}\right)\right\rangle$ as a function of $\tau$; no obvious plateau is discernible even if we go to relatively larger $t_{f}$, which means that the excited-states contaminations are large and, in this case, even a two-state fit cannot extract the ME reliably. To be more specific, all the components of the AWI are plotted in the right panel of Fig. 4. In this $|\vec{q}|=\frac{2 \pi}{L}$ case $\Delta E a \sim 0.03$, so the values of $\Delta E\left\langle A_{4}(\tau, \vec{q})\right\rangle$ are very close to 0 ; however, the $\left\langle\partial_{4} A_{4}\right\rangle$ values are of order 0.1 , which pinpoints the problem. One can ask why the $\left\langle\partial_{4} A_{4}\right\rangle$ case is so different since there should also be some excited-states contamination. The answer is that the AWI is actually a relation of the current operators and it should hold regardless of whether the currents are inserted between two nucleon ground states or excited states. The only problem is that when we use $\left\langle A_{4}(\tau, \vec{q})-A_{4}(\tau-1, \vec{q})\right\rangle=\Delta E\left\langle A_{4}(\tau, \vec{q})\right\rangle$, we are assuming that the $\mathrm{ME}$ is the ground-state $\mathrm{ME}$ and the $\Delta E$ is the energy difference between two ground states which is, apparently, not the case. We can thus conclude that if the conditions $\tau \gg 0$ and $t_{f} \gg \tau$ are satisfied, the three ratios will be the same; for finite $\tau$ and $t_{f}$, the ratio of $R_{2}$ is the preferable check of the AWI. The results of $R_{2}$ show that the AWI is well satisfied in our case and we do not need any extra normalization factor in addition to the isovector one $Z_{A}^{3}$ to make the AWI hold for the DI calculations for all the three lattices and all the quark masses. In other words, we have $Z_{A}^{0}=Z_{A}(\mathrm{CI})=Z_{A}(\mathrm{DI})=Z_{A}^{3}$.

\section{B. CI case}

We also check the chiral Ward identity in the CI case. In fact, the violation of the chiral Ward identity in terms of form factors at small momentum transfers in the CI case is also observed and reported in [32], where their formula is equivalent to checking the ratio $R_{1}$. In the CI case, the definitions of $R_{1}$ and $R_{2}$ are the same as those in the DI case but without the topological charge term. The results of $R_{1}$ and $R_{2}$ at different $t_{f}$ of the 24I lattice are plotted in Fig. 5 as an example. The horizontal line in the figure indicating $Z_{A}^{3}=1.111(6)$ shows where the points of the ratios should be if the chiral Ward identity holds. Again, the points of $R_{1}$ show an obvious discrepancy. But a trend that the points are approaching the horizontal line with larger $t_{f}$ can be observed. As a contrast, the points of $R_{2}$ at both $t_{f}=8 a$ and $t_{f}=10 a$ do lie on the target line except for the boundary points, showing valid Ward identity.

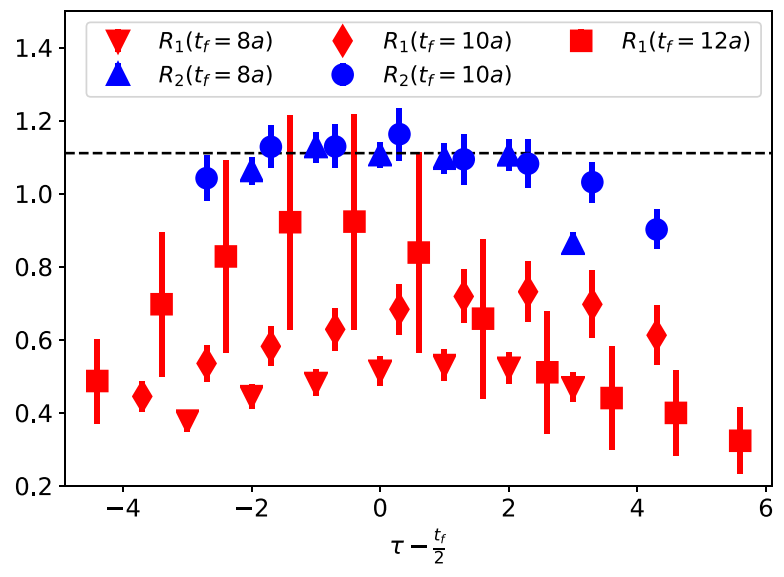

FIG. 5. The ratios of $R_{1}$ and $R_{2}$ in the CI case at different $t_{f}$. The results are from the 24I lattice at the unitary point with momentum transfer $|\vec{q}|=\frac{2 \pi}{L}$. The horizontal line indicating $Z_{A}^{3}=$ 1.111(6) shows that the values of $R_{2}$ are consistent with this normalization factor except for the boundary points. $R_{1}$ shows a large discrepancy and approaches the horizontal line with large $t_{f}$. Points of different $t_{f}$ are shifted slightly to enhance the legibility.

The results of the CI case are similar and the conclusion is the same. The ratio $R_{2}$ shows well established chiral Ward identity while $R_{1}$ shows violation. The difference of $R_{1}$ and $R_{2}$ reflects how we treat the $A_{4}$ term. Even if the form factors $g_{A}\left(q^{2}\right)$ and $h_{A}\left(q^{2}\right)$ are calculated using $A_{i}$ only, the ratio $R_{1}$ is still problematic since Eq. (19) is used in the derivation and it assumes that the $\mathrm{ME}$ of $A_{4}$ gives the same form factors without excited-states contamination. A cure to this problem is to go to large enough $t_{f}$ where the excited-states contamination can be ignored. Unfortunately, this requires much larger statistics. We will test this in the future.

\section{DISCONNECTED-INSERTION CONTRIBUTION}

As is mentioned above, we use the two-state fit to extract the MEs. Examples of the fitting on the 32ID lattice at the unitary point can be found in Fig. 6; both the light and strange quark results are included. We use three source-sink separations $t_{f}=6 a, 7 a$, and $8 a$, which correspond to 0.86 , 1.00 , and $1.15 \mathrm{fm}$, respectively, to carry out the fit. The fitting results are shown by the cyan bands, which are consistent with the data points of largest separation within errors. The contact points on either the source or the sink side are always excluded, and more points may be excluded to have better $\chi^{2}$. A prior value of $\delta m$ is used to stabilize the fits. A criterion used to choose the prior value and width is that the fitting result of $\delta m$ should have statistical significance and the final result of $g_{A}$ should not be too far away from the data points of large separations. The difference of the fitting results due to the choice of prior values is included in the systematic uncertainty. For the 24I and 32I 

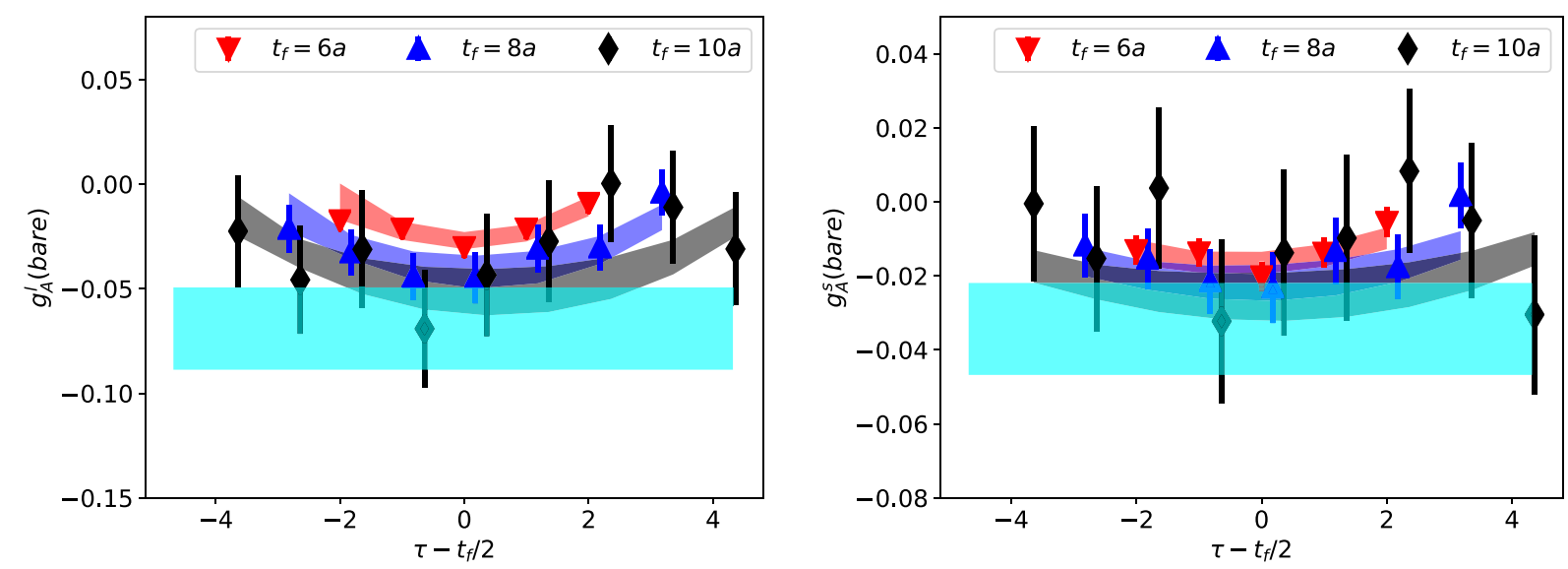

FIG. 6. Examples of two-state fits on the 32ID lattice at the unitary point. The light quark result is on the left while the strange quark result is on the right. Cyan bands are the fitting results from $t_{f}=6 a, 7 a$, and $8 a$, which are consistent with the points of large separations. Points of different $t_{f}$ are shifted slightly in the horizontal direction to enhance the legibility.

lattices, the two-state fits are done similarly. Table III lists all the fitting setups.

The CDER technique [7] is used in order to better control the statistical uncertainties for the 32ID lattice where the CDER technique may improve the signal more significantly since the size of this lattice $L \sim 4.6 \mathrm{fm}$ is relatively large, while we do not use this technique for the 24I and 32I lattices due to their small sizes ( $L \sim 2.7 \mathrm{fm}$ and $L \sim 2.6 \mathrm{fm}$, respectively). In order to use the CDER technique, the three-point functions can be rewritten as

$$
C_{3, \mu}\left(t_{f}, \tau, R\right)=\sum_{\vec{x},|\vec{r}|<R}\left\langle\chi\left(t_{f}, \vec{x}\right) A_{\mu}(\tau, \vec{x}+\vec{r}) \bar{\chi}(0, \mathcal{G})\right\rangle
$$

where we put a cutoff $R$ to the distance between the quark loop and the sink of the nucleon propagator and we can vary $R$ to obtain different three-point functions. As demonstrated in [7], the signal will saturate after $R$ is larger than the corresponding correlation length, but the noise will keep growing due to the fact that the variances of the two disconnected operators in their vacuum expectation values are independent of each other. Therefore an optimal cutoff

TABLE III. Setups of the two-state fits in the DI case. The source-sink separations used in the fits, the number of points dropped on the source side, the number of points dropped on the sink side, and the prior value and width of $\delta m$ are listed for each lattice and for both light and strange flavors.

\begin{tabular}{lcccc}
\hline \hline Lattice/flavor & Separations (a) & $\begin{array}{c}\text { Source } \\
\text { drop }\end{array}$ & $\begin{array}{c}\text { Sink } \\
\text { drop }\end{array}$ & $\begin{array}{c}\text { Prior } \\
\delta m a\end{array}$ \\
\hline $32 \mathrm{ID} / l$ & $6,7,8$ & 2 & 2 & $0.4(0.1)$ \\
$32 \mathrm{ID} / s$ & $6,7,8$ & 1 & 1 & $0.3(0.1)$ \\
$24 \mathrm{I} / l$ & $8,9,10,11$ & 2 & 2 & $0.4(0.1)$ \\
$24 \mathrm{I} / s$ & $8,9,10,11$ & 2 & 1 & $0.3(0.2)$ \\
$32 \mathrm{I} / l$ & $9,10,11,12$ & 3 & 2 & $0.4(0.1)$ \\
$32 \mathrm{I} / s$ & $9,10,11,12$ & 3 & 2 & $0.3(0.2)$ \\
\hline \hline
\end{tabular}

$R$ can be found if the lattice size is larger than the correlation length between the operators whereupon the signal-to-noise ratio is improved. However, as is shown in Fig. 7 where the $g_{A}(\mathrm{DI})$ from Eq. (29) is plotted as a function of $R$, no clear plateau shows until at very large $R$, especially for the light quark case, which is probably because the correlation length is not much smaller than the lattice size. So we cannot find an optimal $R$ in this case. Nevertheless, a correlated fit using the following asymptotic form [7]:

$$
C_{3}(R)=C_{3}(\infty)+k \sqrt{R} \frac{e^{-M R}}{M}
$$

with $k$ and $M$ being free parameters helps in extracting $C_{3}(\infty)$ properly. The blue bands in Fig. 7 show the results of the correlated fit while the green bands show the results of an uncorrelated fit in contrast. The reason why we need this comparison is because the data points of different $R$ are strongly correlated and an uncorrelated fit will underestimate the error very much. To keep the correlations of different $R$, we cannot do single two-state fits, respectively, for each $R$. Instead, a simultaneous two-state fit combining all the $R$ to keep the whole correlation matrix is carried out. The error of the correlated fit, which is not much smaller than the error of the data points with large cutoff, is believed to be a reasonable estimation. In this way, the final statistical uncertainties of the MEs on the 32ID lattice can be reduced by $10 \%-40 \%$ for different quark masses.

\section{CONNECTED-INSERTION CONTRIBUTION}

As for the CI case, we use the improved axial-vector current following our previous work on the 24I and 32I lattices [26] to reduce the discretization errors on the 32ID lattice as well. For the 24I and 32I lattices, we reanalyze the data with the $u$ quark and the $d$ quark separately. Two-state 

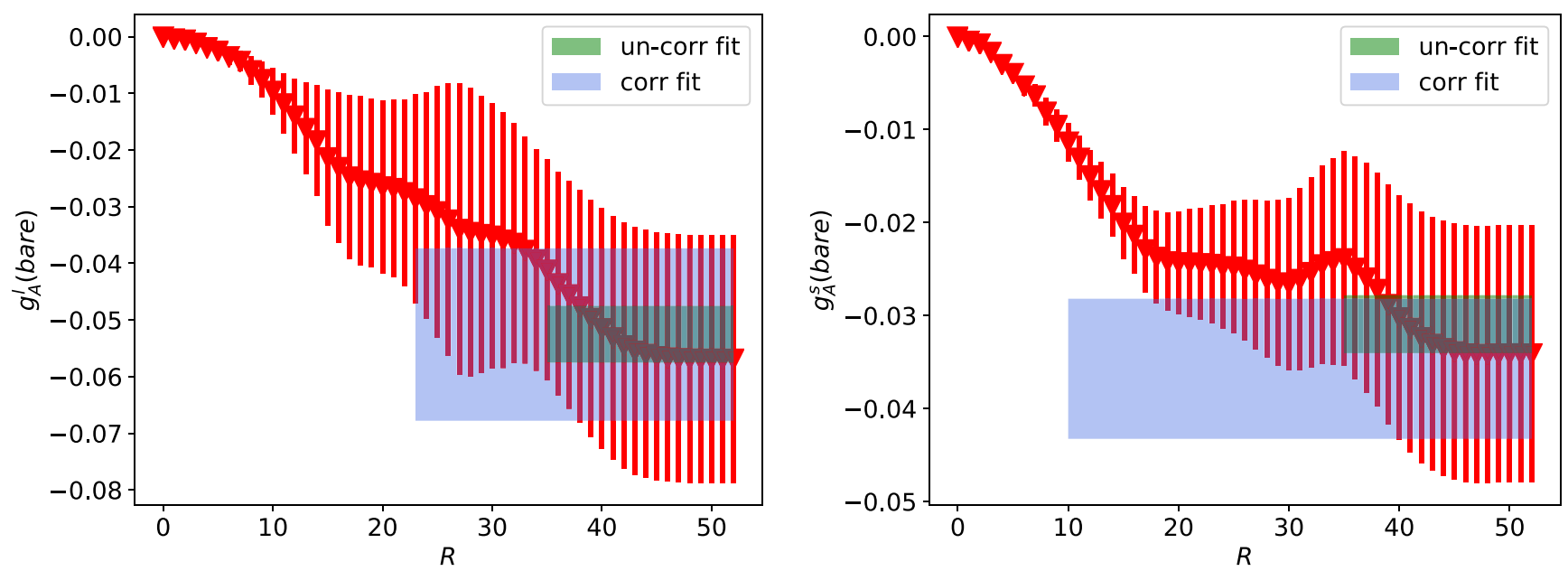

FIG. 7. The $R$ dependence of the axial coupling of the 32ID lattice at the unitary point. The left panel is for the light quark case, and the right panel is for the strange quark case. Blue bands and green bands are for correlated fit and uncorrelated fit, respectively.

fits are also applied to the MEs of $A_{i}=\bar{\psi} i \gamma_{i} \gamma_{5} \hat{\psi}$, and the fitting setups are also listed in Table IV. We plot the fitting results of the 32ID lattice at the unitary point in Fig. 8 as an example. To implement the improvement, we also need to fit for the MEs of three more currents: $A_{4}=\bar{\psi} i \gamma_{5} \gamma_{5} \hat{\psi}$, $D_{i}=\bar{\psi} i \sigma_{i \mu} \stackrel{\leftrightarrow}{D}_{\mu} \gamma_{5} \hat{\psi}$, and $D_{4}=\bar{\psi} i \sigma_{4 i} \stackrel{\leftrightarrow}{D}_{i} \gamma_{5} \hat{\psi}$. For these currents, the signal-to-noise ratio is not as good as that for the $A_{i}$ case, and no obvious excited-state contribution can be observed; we are only able to make a constant fit combining several separations. An example of $D_{4}$ is plotted in Fig. 9; note that we drop three points on each of the source and sink sides.

The spatial and temporal components of the improved axial-vector current are defined as $A_{i}^{\mathrm{im}}=A_{i}+g D_{i}$ and $A_{4}^{\mathrm{im}}=A_{4}+g D_{4}$, where the factor $g$ is determined by assuming the final $g_{A}$ calculated from the two components of the improved current are identical [26]. Although the results of the currents with derivatives are noisy and the constant fit may not be a perfect choice, it is enough for this calculation since the improvement itself is only around 3\% or less. Plots of the improvement are shown in Fig. 10. For

TABLE IV. Setups of the two-state fits in the CI case for the current $A_{i}=\bar{\psi} \gamma_{5} \gamma_{i} \hat{\psi}$. The source-sink separations used in the fits, the number of points dropped on the source side, the number of points dropped on the sink side, and the prior value and width of $\delta m$ are listed for each lattice and for both $u$ and $d$ quarks.

\begin{tabular}{lcccc}
\hline \hline Lattice/flavor & Separations (a) & $\begin{array}{c}\text { Source } \\
\text { drop }\end{array}$ & $\begin{array}{c}\text { Sink } \\
\text { drop }\end{array}$ & $\begin{array}{c}\text { Prior } \\
\delta m a\end{array}$ \\
\hline $32 \mathrm{ID} / u$ & $7,8,9,10,11$ & 3 & 2 & $0.35(0.1)$ \\
$32 \mathrm{ID} / d$ & $7,8,9,10,11$ & 2 & 2 & $0.35(0.1)$ \\
$24 \mathrm{I} / u$ & $8,10,11,12$ & 2 & 1 & $0.3(0.1)$ \\
$24 \mathrm{I} / d$ & $8,10,11,12$ & 3 & 1 & $0.3(0.1)$ \\
$32 \mathrm{I} / u$ & $12,14,15$ & 2 & 2 & $0.2(0.1)$ \\
$32 \mathrm{I} / d$ & $12,14,15$ & 4 & 3 & $\cdots$ \\
\hline \hline
\end{tabular}

the $d$ quark case, the improvement has no effect basically, while for the $u$ quark case, especially for the 24I lattice, the improvement is at the $2 \sigma$ level and the improved data points are closer to the points of the other two lattices around a similar pion mass, manifesting smaller lattice spacing effects.

\section{RENORMALIZATION}

The renormalization of the axial-vector current is indispensable for comparing our result with experiment and phenomenology. The scale-independent isovector normalization constant $Z_{A}(\mathrm{CI})$ can be calculated by imposing the chiral Ward identity in CI as in Eq. (13) or between the vacuum and a pion state [31]. There is no difference between the $u$ and $d$ quark in this case, as can be seen in the $\mathrm{RI} / \mathrm{MOM}$ nonperturbative procedure. Hence, $Z_{A}(\mathrm{CI})=Z_{A}^{3}$, the isovector normalization constant. Since we adopt the mass-independent renormalization scheme, it is also the same as the octet renormalization $Z_{A}^{8}$. We shall define $Z_{A} \equiv$ $Z_{A}^{3}=Z_{A}^{8}$ as conventionally used in the literature. After checking the AWI in the DI, we concluded in Sec. IV that the axial-vector current with the normalization of $Z_{A}(\mathrm{CI})$ satisfies the AWI; thus there is no additional normalization factor for the AWI, and $Z_{A}(\mathrm{DI})=Z_{A}(\mathrm{CI})=Z_{A}$ is the only normalization constant as far as tree-level AWI is concerned. Through the chiral Ward identity in the CI, we can determine $Z_{A}$ to a high precision. Since we have calculated MEs of both CI and DI, the disconnected part of vertex functions also needs to be computed and the corresponding renormalization can be obtained by the lattice nonperturbative approach in the RI/MOM scheme [33]. This part contains a scale-dependent DI piece and also mixing effects and is referred to as renormalization, to be distinguished from the normalization, discussed so far, in upholding the AWI at the tree level. 

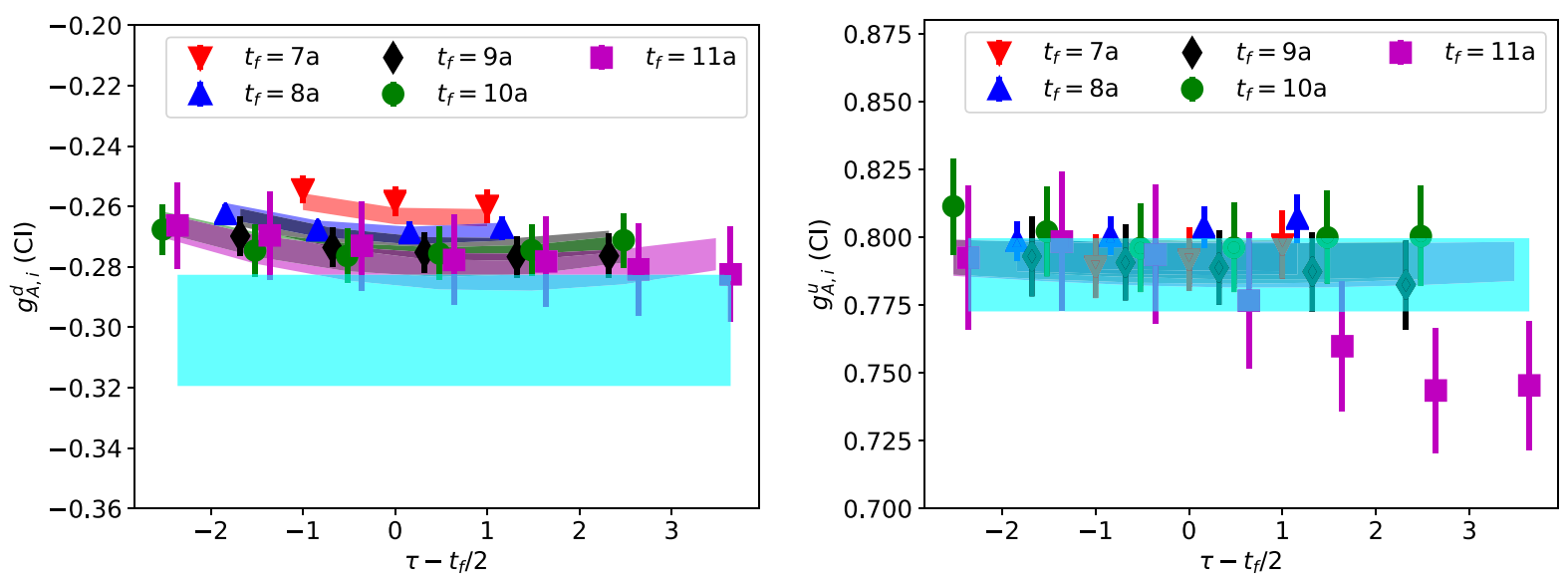

FIG. 8. Two-state fit examples for the $d$ quark and the $u$ quark, respectively, on the 32ID lattice. The labels $g_{A, i}^{d}$ and $g_{A, i}^{u}$ denote the axial couplings for $d$ and $u$ quarks calculated from current $A_{i}=\bar{\psi} i \gamma_{i} \gamma_{5} \hat{\psi}$. Points of different $t_{f}$ are shifted slightly in the horizontal direction to enhance the legibility.

\section{A. Formalisms}

The axial-vector coupling has conventionally been classified as the isovector $g_{A}^{3}=\Delta u-\Delta d$, the octet $g_{A}^{8}=\Delta u+$ $\Delta d-2 \Delta s$ through the diagonal $S U(3)$ chiral transformation, and the singlet $g_{A}^{0}=\Delta u+\Delta d+\Delta s$ through the $\mathrm{U}(1)$ transformation, and their renormalization follows. One can obtain the renormalized $\Delta u, \Delta d$, and $\Delta s$ in term of their unrenormalized counterparts through these flavorirreducible representations, and the details are given in Ref. [34]. On the other hand, the lattice calculations are carried out in terms of flavors and MEs in the CI and DI. It is natural to use them as the basis in renormalization. As we shall see, this has the advantage of preserving the CI piece that is scale independent and can be compared in different lattice calculations. Moreover, it is physical and can be extracted from the global fitting of the polarized parton distribution function (PDF).

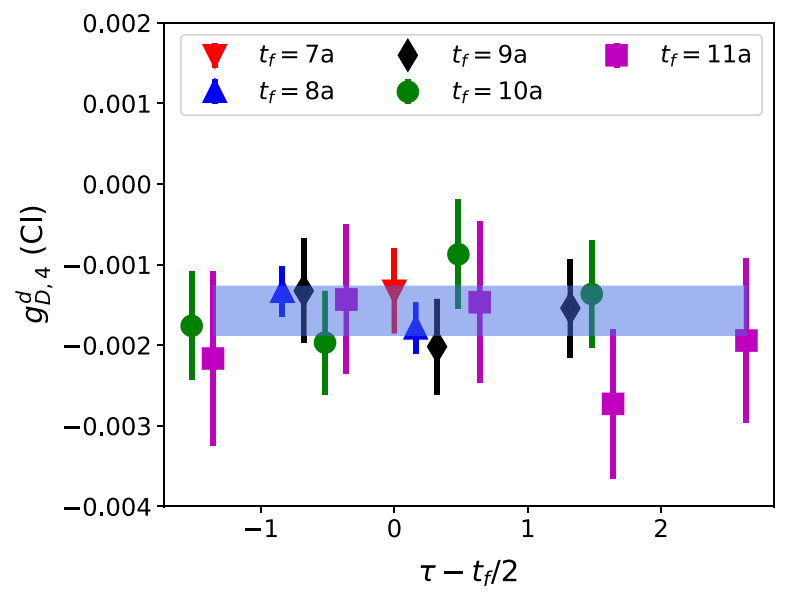

FIG. 9. Constant fit example for the $d$ quark case of current $D_{4}$. Points of different $t_{f}$ are shifted slightly in the horizontal direction to enhance the legibility.
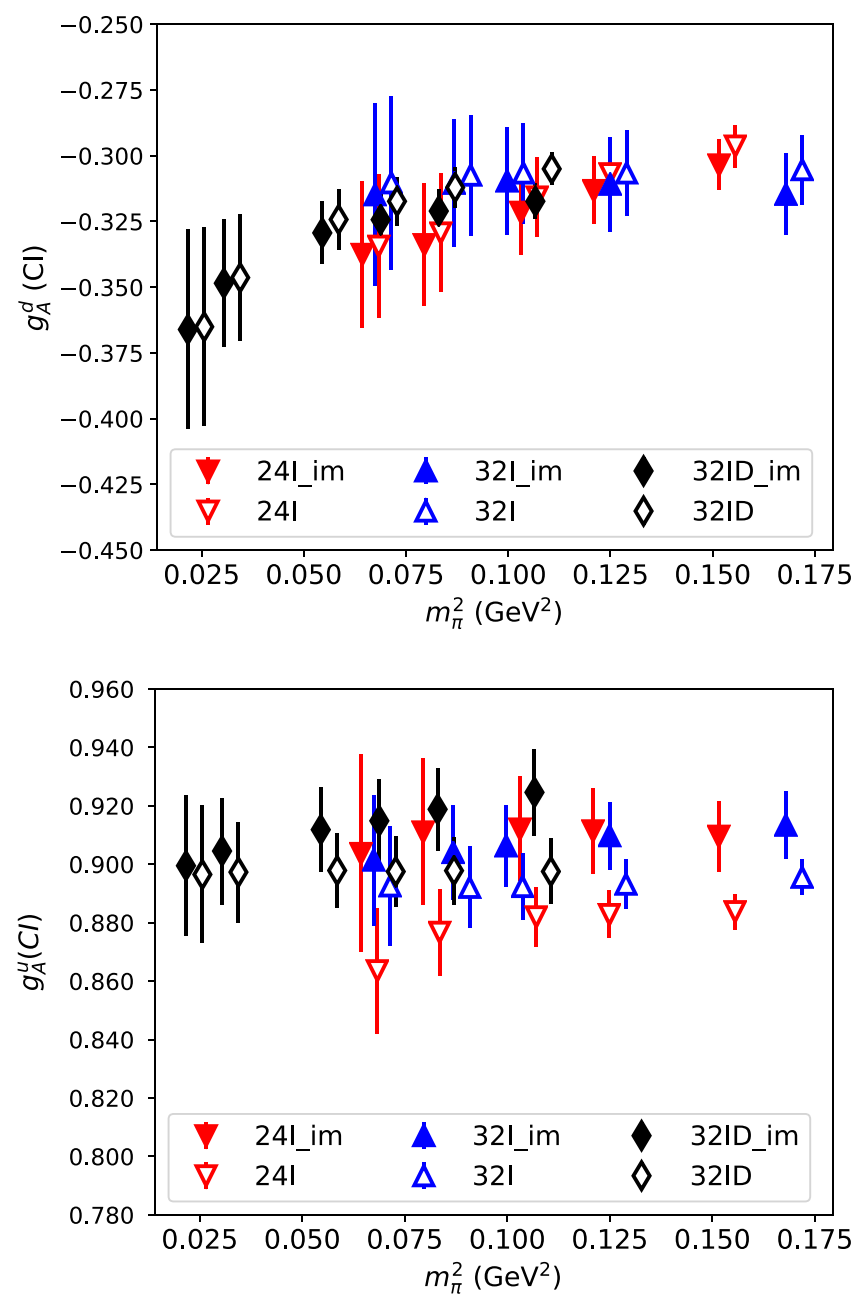

FIG. 10. The comparison of the MEs before and after the improvement as a function of pion mass squared. The top panel is for the $d$ quark and the bottom panel is for the $u$ quark. Points of the unimproved results are shifted slightly in the horizontal direction to enhance the legibility. 
In the RI/MOM renormalization scheme, the renormalized quantities are related to the unrenormalized ones through the vertex and the field renormalization. The most general form from the lattice classification is the following:

$$
\left(\begin{array}{c}
\Delta u(\mathrm{CI}) \\
\Delta d(\mathrm{CI}) \\
\Delta u(\mathrm{DI}) \\
\Delta d(\mathrm{DI}) \\
\Delta s(\mathrm{DI})
\end{array}\right)=\left(\begin{array}{ccccc}
\Sigma_{C} & 0 & 0 & 0 & 0 \\
0 & \Sigma_{C} & 0 & 0 & 0 \\
\Sigma_{D} & \Sigma_{D} & \Sigma_{C}+\Sigma_{D} & \Sigma_{D} & \Sigma_{D} \\
\Sigma_{D} & \Sigma_{D} & \Sigma_{D} & \Sigma_{C}+\Sigma_{D} & \Sigma_{D} \\
\Sigma_{D} & \Sigma_{D} & \Sigma_{C} & \Sigma_{C} & \Sigma_{C}+\Sigma_{D}
\end{array}\right)\left(\begin{array}{c}
\Delta u^{\mathrm{RI}}(\mathrm{CI}) \\
\Delta d^{\mathrm{RI}}(\mathrm{CI}) \\
\Delta u^{\mathrm{RI}}(\mathrm{DI}) \\
\Delta d^{\mathrm{RI}}(\mathrm{DI}) \\
\Delta s^{\mathrm{RI}}(\mathrm{DI})
\end{array}\right)
$$

where $\Delta f(f=u, d, s)$ is the bare axial-vector current matrix element for a particular flavor $f$ and $\Delta f^{\mathrm{RI}}$ is the corresponding renormalized one in the RI scheme. $\Sigma_{C}$ or $\Sigma_{D}$ in the matrix is defined by the following trace indicating the renormalization condition:

$$
\Sigma_{C / D}=Z_{q}^{-1} \frac{1}{12} \operatorname{Tr}\left[\Lambda_{C / D}(p) \Lambda^{\text {tree }}(p)^{-1}\right],
$$

where $Z_{q}$ is the quark field renormalization constant, $\Lambda_{C / D}(p)$ is the connected or disconnected part of the vertex function, and $\Lambda^{\text {tree }}(p)$ is the tree-level vertex. The vertex function $\Lambda_{C / D}(p)$ is the following amputated Green's function:

$$
\Lambda_{C / D}(p)=S^{-1}(p) G_{A, C / D}(p) S^{-1}(p),
$$

where $S^{-1}(p)$ is a quark propagator in the momentum space in the Landau gauge and $G_{A, C / D}(p)$ is the connected piece or the disconnected piece of the forward Green's function $G_{A}(p)=\sum_{x, y} e^{-i p \cdot(x-y)}\left\langle\psi(x) \Gamma_{A} \bar{\psi}(y)\right\rangle$ with $\Gamma_{A}=\gamma_{\mu} \gamma_{5}$. To be more specific, the two ways of Wick contraction of $G_{A}(p)$ lead to two kinds of the vertex function that are the connected part $\Lambda_{C}$, where the quark fields in the bilinear

$$
\left(\begin{array}{c}
\Delta u^{\mathrm{RI}}(\mathrm{CI}) \\
\Delta d^{\mathrm{RI}}(\mathrm{CI}) \\
\Delta u^{\mathrm{RI}}(\mathrm{DI}) \\
\Delta d^{\mathrm{RI}}(\mathrm{DI}) \\
\Delta s^{\mathrm{RI}}(\mathrm{DI})
\end{array}\right)=\left(\begin{array}{ccc}
Z_{A} & 0 & 0 \\
0 & Z_{A} & 0 \\
Z_{A}^{\mathrm{D}, \mathrm{RI}} & Z_{A}^{\mathrm{D}, \mathrm{RI}} & Z_{A}+Z_{A}^{\mathrm{D}, \mathrm{RI}} \\
Z_{A}^{\mathrm{D}, \mathrm{RI}} & Z_{A}^{\mathrm{D}, \mathrm{RI}} & Z_{A}^{\mathrm{D}, \mathrm{RI}} \\
Z_{A}^{\mathrm{D}, \mathrm{RI}} & Z_{A}^{\mathrm{D}, \mathrm{RI}} & Z_{A}^{\mathrm{D}, \mathrm{RI}}
\end{array}\right.
$$

where

$$
Z_{A}^{\mathrm{D}, \mathrm{RI}} \equiv-Z_{A}\left(\frac{\Sigma_{D}}{\Sigma_{C}+N_{f} \Sigma_{D}}\right), \quad Z_{A}=\frac{1}{\Sigma_{C}} .
$$

In the present calculation, $N_{f}=3$.

To compare with experiments, we need to match the results from the above RI scheme to that of $\overline{\mathrm{MS}}$ at $2 \mathrm{GeV}$. As we see from Sec. II, it entails a two-loop perturbative operator contract with the other two external quark fields, and the disconnected one $\Lambda_{D}$, where the quark fields in the bilinear operator contract with each other. Since only in $\Lambda_{D}$ can the flavor of the bilinear operator be different from that of the external legs, the off-diagonal entries of the matrix in Eq. (31) that represent the flavor mixing effect contain $\Sigma_{D}$ alone. We should stress that the entries of zero reflect the fact that the CI MEs do not receive mixing from the DIs. On the other hand, the DI MEs receive contributions from both $\mathrm{CI}$ and DI. These equations are defined in the quark massless limit so that the RI-MOM is a mass-independent renormalization scheme. In practice, we do calculations at finite quark masses and then extrapolate to the chiral limit. In principle, $Z_{q}$ can be determined by considering the derivative of the quark propagator with respect to the discretized momenta. However, $Z_{q}$ so determined is known to have a large discretization error. We shall use $Z_{A}$ from the chiral Ward identity as an input; therefore, we have $\Sigma_{C}=\frac{1}{Z_{A}}$ and $Z_{q}$ is determined via Eq. (32) instead as employed in Ref. [31].

The renormalization constants come from the inverse of the matrix in Eq. (31). The renormalized quark spins in the RI scheme are

$$
\left.\begin{array}{cc}
0 & 0 \\
0 & 0 \\
Z_{A}^{\mathrm{D}, \mathrm{RI}} & Z_{A}^{\mathrm{D}, \mathrm{RI}} \\
Z_{A}+Z_{A}^{\mathrm{D}, \mathrm{RI}} & Z_{A}^{\mathrm{D}, \mathrm{RI}} \\
Z_{A}^{\mathrm{D}, \mathrm{RI}} & Z_{A}+Z_{A}^{\mathrm{D}, \mathrm{RI}}
\end{array}\right)\left(\begin{array}{c}
\Delta u(\mathrm{CI}) \\
\Delta d(\mathrm{CI}) \\
\Delta u(\mathrm{DI}) \\
\Delta d(\mathrm{DI}) \\
\Delta s(\mathrm{DI})
\end{array}\right)
$$

calculation of the axial-vector current in the RI and $\overline{\mathrm{MS}}$ schemes, respectively. On the other hand, as it is shown in Eq. (7), this is the same renormalization constant from the one-loop mixing of the topological charge. We carry out the simpler one-loop mixing calculation of the topological charge for the matching factor from the RI scheme at momentum $p$ to the $\overline{\mathrm{MS}}$ scheme at scale $\mu$ based on PACKAGE-X $[35,36]$, and this matching ratio can be represented as a matrix $R_{m}$ that needs to be 


$$
R_{m}=\left(\begin{array}{ccccc}
1 & 0 & 0 & 0 & 0 \\
0 & 1 & 0 & 0 & 0 \\
f_{m} & f_{m} & 1+f_{m} & f_{m} & f_{m} \\
f_{m} & f_{m} & f_{m} & 1+f_{m} & f_{m} \\
f_{m} & f_{m} & f_{m} & f_{m} & 1+f_{m}
\end{array}\right)
$$

where $f_{m}=\left(\frac{\alpha_{s}}{4 \pi}\right)^{2} 4 C_{F}\left(-\frac{3}{2} \log \left(\frac{\mu^{2}}{p^{2}}\right)+\frac{7}{2}\right)$ with $C_{F}=4 / 3$.

Thus, after $R_{m}$ is multiplied to the renormalization matrix in Eq. (34), the renormalized quark spin in the $\overline{\mathrm{MS}}$ scheme is

$$
\left(\begin{array}{c}
\Delta u^{N}(\mathrm{CI}) \\
\Delta d^{N}(\mathrm{CI}) \\
\Delta u^{\overline{\mathrm{MS}}}(\mathrm{DI})(\mu) \\
\Delta d^{\overline{\mathrm{MS}}}(\mathrm{DI})(\mu) \\
\Delta s^{\overline{\mathrm{MS}}}(\mathrm{DI})(\mu)
\end{array}\right)=\left(\begin{array}{ccccc}
Z_{A} & 0 & 0 & 0 & 0 \\
0 & Z_{A} & 0 & 0 & 0 \\
Z_{A}^{\mathrm{D}, \overline{\mathrm{MS}}} & Z_{A}^{\mathrm{D}, \overline{\mathrm{MS}}} & Z_{A}+Z_{A}^{\mathrm{D}, \overline{\mathrm{MS}}} & Z_{A}^{\mathrm{D}, \overline{\mathrm{MS}}} & Z_{A}^{\mathrm{D}, \overline{\mathrm{MS}}} \\
Z_{A}^{\mathrm{D}, \overline{\mathrm{MS}}} & Z_{A}^{\mathrm{D}, \overline{\mathrm{MS}}} & Z_{A}^{\mathrm{D}, \overline{\mathrm{MS}}} & Z_{A}+Z_{A}^{\mathrm{D}, \overline{\mathrm{MS}}} & Z_{A}^{\mathrm{D}, \overline{\mathrm{MS}}} \\
Z_{A}^{\mathrm{D}, \overline{\mathrm{MS}}} & Z_{A}^{\mathrm{D}, \overline{\mathrm{MS}}} & Z_{A}^{\mathrm{D}, \overline{\mathrm{MS}}} & Z_{A}^{\mathrm{D}, \overline{\mathrm{MS}}} & Z_{A}+Z_{A}^{\mathrm{D}, \overline{\mathrm{MS}}}
\end{array}\right)\left(\begin{array}{c}
\Delta u(\mathrm{CI}) \\
\Delta d(\mathrm{CI}) \\
\Delta u(\mathrm{DI}) \\
\Delta d(\mathrm{DI}) \\
\Delta s(\mathrm{DI})
\end{array}\right),
$$

where the notations of $\Delta u^{N}(\mathrm{CI})$ and $\Delta d^{N}(\mathrm{CI})$ mean they have normalization only and

$$
Z_{A}^{\mathrm{D}, \overline{\mathrm{MS}}}=Z_{A}^{\mathrm{D}, \mathrm{RI}}+f_{m}+N_{f} f_{m} Z_{A}^{\mathrm{D}, \mathrm{RI}} .
$$

In practice, $Z_{A}^{\mathrm{D}, \overline{\mathrm{MS}}}$ are to be evolved to a given scale such as $2 \mathrm{GeV}$ for each $p^{2} a^{2}$ in RI and extrapolated to $p^{2} a^{2}=0$. This involves an evolution

$$
\mu^{2} \frac{d}{d \mu^{2}} \log \left(Z_{A}^{\mathrm{D}, \overline{\mathrm{MS}}}(\mu)\right)=\gamma\left(\alpha_{s}\right)=-\sum_{i} \gamma_{i} \alpha_{s}^{i+1}
$$

For the axial-vector current, the anomalous dimensions are

$$
\gamma_{0}=0, \quad \gamma_{1}=\frac{1}{(4 \pi)^{2}} 6 C_{F} N_{f}
$$

It is shown in [34] that, at two-loop order, the evolution of the flavor-singlet renormalization factor is given by

$$
\frac{Z_{A}+3 Z_{A}^{\mathrm{D}, \overline{\mathrm{MS}}}(\mu)}{Z_{A}+3 Z_{A}^{\mathrm{D}, \overline{\mathrm{MS}}}\left(\mu_{0}\right)}=\left(\frac{\beta_{0}+\beta_{1} \alpha_{s}(\mu)}{\beta_{0}+\beta_{1} \alpha_{s}\left(\mu_{0}\right)}\right)^{\gamma_{1} / \beta_{1}},
$$

where $Z_{A}+3 Z_{A}^{\mathrm{D}, \overline{\mathrm{MS}}}(\mu)$ is the renormalization constant for the flavor-singlet case which we will show later, and the relevant constants are $\beta_{0}=\frac{1}{4 \pi}\left(\frac{11}{3} C_{A}-\frac{4}{3} T_{F} N_{f}\right)=\frac{1}{4 \pi}\left(11-\frac{2}{3} N_{f}\right)$ and $\quad \beta_{1}=\frac{1}{(4 \pi)^{2}}\left(\frac{34}{3} C_{A}^{2}-\frac{20}{3} C_{A} T_{F} N_{f}-4 C_{F} T_{F} N_{f}\right)=$ $\frac{1}{(4 \pi)^{2}}\left(102-\frac{38}{3} N_{f}\right)$ with $C_{A}=3$ and $T_{F}=\frac{1}{2}$. The evolution of $\alpha_{s}$ at the two-loop level is given in [37] $\alpha_{s}(\mu)=-\frac{\beta_{0}}{\beta_{1}} \frac{1}{1+W_{-1}(\zeta)}, \quad \zeta=-\frac{\beta_{0}^{2}}{e \beta_{1}}\left(\frac{\Lambda^{2}}{\mu^{2}}\right)^{\beta_{0}^{2} / \beta_{1}}$,

where $W_{-1}$ is the lower branch of the Lambert function and $\Lambda$ is set to be the particle data group (PDG) value 332(19) $\mathrm{MeV}$.

The final results of the renormalized $u / d$ quark spin can be decomposed into the CI part and the DI part for each flavor

$(\Delta u / \Delta d)^{\overline{\mathrm{MS}}}(\mu)=(\Delta u / \Delta d)^{N}(\mathrm{CI})+(\Delta u / \Delta d)^{\overline{\mathrm{MS}}}(\mathrm{DI})(\mu)$,

where the connected insertion part

$$
(\Delta u / \Delta d)^{N}(\mathrm{CI})=Z_{A}(\Delta u / \Delta d)(\mathrm{CI})
$$

is scale independent. We should caution that this is true for the axial-vector case due to the chiral Ward identity. This is not true in general, such as for the case of the scalar and the energy-momentum tensor matrix elements where the CI parts are also scale dependent. On the other hand, the disconnected insertion parts depend on the $\overline{\mathrm{MS}}$ scale of $\mu$,

$$
\begin{aligned}
(\Delta u / \Delta d)^{\overline{\mathrm{MS}}}(\mathrm{DI})(\mu) & =Z_{A}(\Delta u / \Delta d)(\mathrm{DI})+Z_{A}^{\mathrm{D}, \overline{\mathrm{MS}}}(\mu) \Sigma, \\
\Delta s^{\overline{\mathrm{MS}}}(\mu) & =Z_{A} \Delta s+Z_{A}^{\mathrm{D}, \overline{\mathrm{MS}}}(\mu) \Sigma,
\end{aligned}
$$

where 


$$
\begin{aligned}
\Sigma= & \Delta u+\Delta d+\Delta s=\Delta u(\mathrm{CI})+\Delta d(\mathrm{CI}) \\
& +(\Delta u+\Delta u+\Delta s)(\mathrm{DI}) .
\end{aligned}
$$

This decomposition of the quark spin in terms of flavor, $\mathrm{CI}$, and DI is common for all renormalization schemes, and is not limited to the RI or $\overline{\mathrm{MS}}$ scheme. When the $\mathrm{CI}$ and DI components are added together from Eq. (37) to get the total matrix elements, one arrives at a simpler expression

$$
\Delta f^{\overline{\mathrm{MS}}}(\mu)=Z_{A} \Delta f+Z_{A}^{\mathrm{D}, \overline{\mathrm{MS}}}(\mu) \Sigma,
$$

where $f=u, d, s$. In terms of the flavor irreducible representations, they are

$$
\begin{gathered}
g_{A}^{3}=\Delta u^{\overline{\mathrm{MS}}}-\Delta d^{\overline{\mathrm{MS}}}=Z_{A}(\Delta u-\Delta d), \\
g_{A}^{8}=\Delta u^{\overline{\mathrm{MS}}}+\Delta d^{\overline{\mathrm{MS}}}-2 \Delta s^{\overline{\mathrm{MS}}}=Z_{A}(\Delta u+\Delta d-2 \Delta s), \\
g_{A}^{0, \overline{\mathrm{MS}}}(\mu)=\Delta u^{\overline{\mathrm{MS}}}+\Delta d^{\overline{\mathrm{MS}}}+\Delta s^{\overline{\mathrm{MS}}}=\left(Z_{A}+3 Z_{A}^{\mathrm{D}, \overline{\mathrm{MS}}}(\mu)\right) \Sigma .
\end{gathered}
$$

Equation (47) can be derived by starting the renormalization from the combined CI and DI matrix elements so that Eq. (37) becomes

$$
\left(\begin{array}{c}
\Delta u^{\overline{\mathrm{MS}}}(\mu) \\
\Delta d^{\overline{\mathrm{MS}}}(\mu) \\
\Delta s^{\overline{\mathrm{MS}}}(\mu)
\end{array}\right)=\left(\begin{array}{ccc}
Z_{A}+Z_{A}^{\mathrm{D}, \overline{\mathrm{MS}}}(\mu) & Z_{A}^{\mathrm{D}, \overline{\mathrm{MS}}}(\mu) & Z_{A}^{\mathrm{D}, \overline{\mathrm{MS}}}(\mu) \\
Z_{A}^{\mathrm{D}, \overline{\mathrm{MS}}}(\mu) & Z_{A}+Z_{A}^{\mathrm{D}, \overline{\mathrm{MS}}}(\mu) & Z_{A}^{\mathrm{D}, \overline{\mathrm{MS}}}(\mu) \\
Z_{A}^{\mathrm{D}, \overline{\mathrm{MS}}}(\mu) & Z_{A}^{\mathrm{D}, \overline{\mathrm{MS}}}(\mu) & Z_{A}+Z_{A}^{\mathrm{D}, \overline{\mathrm{MS}}}(\mu)
\end{array}\right)\left(\begin{array}{c}
\Delta u \\
\Delta d \\
\Delta s
\end{array}\right)
$$

Similarly, Eq. (47) for the renormalized quark spin for each flavor can be derived from the basis of flavors irreducible representations $g_{A}^{3}, g_{A}^{8}$, and $g_{A}^{0}$,

$$
\left(\begin{array}{c}
g_{A}^{3} \\
g_{A}^{8} \\
g_{A}^{0, \overline{\mathrm{MS}}}(\mu)
\end{array}\right)=\left(\begin{array}{ccc}
Z_{A} & 0 & 0 \\
0 & Z_{A} & 0 \\
0 & 0 & Z_{A}+N_{f} Z_{A}^{\mathrm{D}, \overline{\mathrm{MS}}}(\mu)
\end{array}\right)\left(\begin{array}{c}
\Delta u-\Delta d \\
\Delta u+\Delta d-2 \Delta s \\
\Delta u+\Delta d+\Delta s
\end{array}\right)
$$

This has been worked out in [34] with the same results.

It is not surprising that one arrives at the same renormalized results in Eq. (47) irrespective of the starting basis in Eq. (37), (51), or (52), since they involve linear equations. Equation (52) is the conventional way of presenting the renormalized results in both experiments and phenomenology. However, we should stress that there are advantages of separating them further in terms of CI and DI parts as in Eqs. (43)-(45) for each flavor. First of all, we note that the CI parts are renormalization group invariant due to the chiral Ward identity, and they are easier to calculate on the lattice than those of the DI parts so that they can be readily compared from lattice calculations involving different systematics owing to different actions and lattice parameters. More importantly, they can be deduced from experiments. The parton degrees of freedom of the nucleon structure functions in the DIS have been classified in the Euclidean path-integral formalism of the hadronic tensor $[38,39]$. It is found that there is a connected-sea parton that is in the connected insertion of the current-current correlator in addition to the disconnected-sea partons in the corresponding disconnected insertion. The former is responsible for the Gottfried sum-rule violation [38]. These two sea partons have not been separated in the global fittings so far. However, it is demonstrated, in one example, that by combining the strange parton distribution from the semiinclusive DIS experiment of HERMES, the global fitting result of $\bar{u}(x)+\bar{d}(x)$, and the lattice calculation of the ratio of $\frac{\langle x\rangle_{s}}{\langle x\rangle_{u}(\mathrm{DI})}$, one can separate the connected-sea from the disconnected-sea distribution of the $u$ and $d$ partons [40]. It is shown that in the operator product expansion, it is the moments of the combined connected-sea and valence parton distributions that correspond to the local matrix elements of the CI in the lattice calculation [39]. The parton evolution equations with separate connected- and disconected-sea partons are formulated [41]. Provided that future global fitting takes this separation into account when fitting experiments at different $Q^{2}$, one can obtain the moments of the valence and connected sea to extract $\Delta u(\mathrm{CI}), \Delta d(\mathrm{CI})$, and other moments from the unpolarized and polarized partons and compare directly with the lattice calculation of moments. 

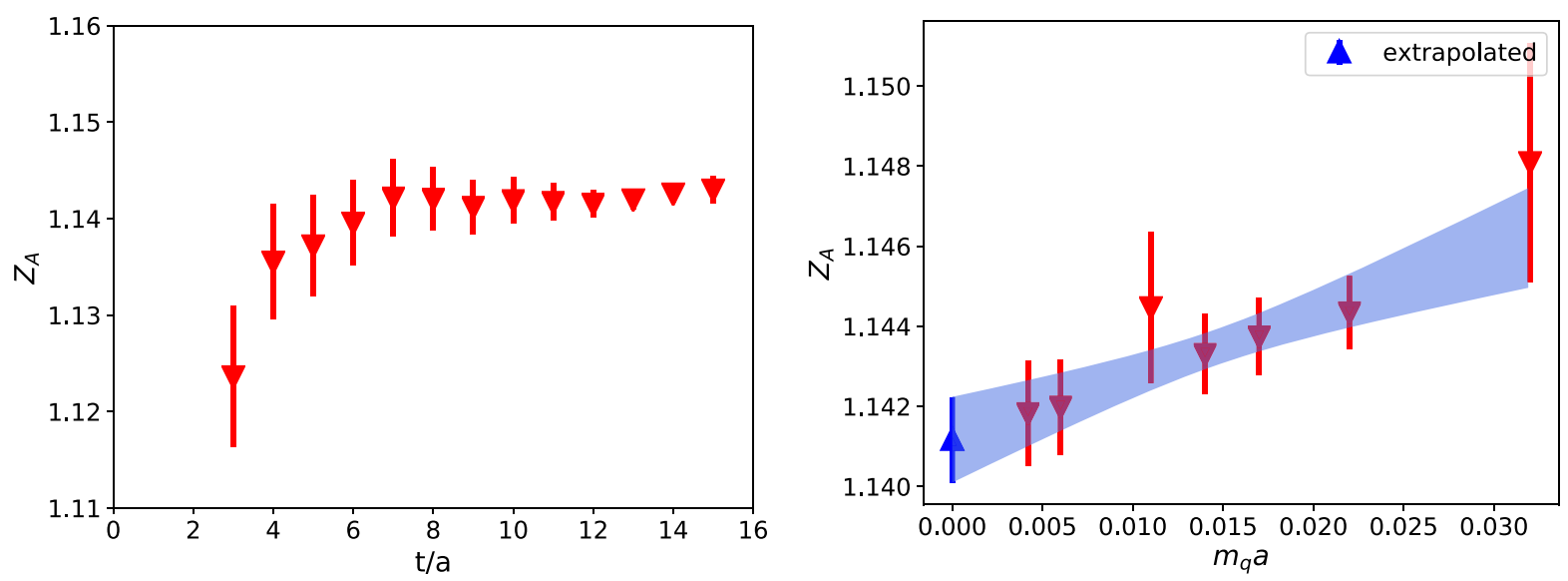

FIG. 11. $Z_{A}$ on the 32ID lattice at the unitary point as a function of $t$ is shown in the left panel. The corresponding chiral extrapolation is shown in the right panel.

\section{B. Numerical results of the renormalization}

The results of $Z_{A}$ on the 24I and 32I lattices have been obtained in our previous study [31] to be 1.111(6) and 1.086(2) at the massless limit for both valence and sea quarks. The $Z_{A}$ on the 32ID lattice is calculated in this study using the same strategy: $Z_{A}=\frac{2 m_{q}\langle 0|P| \pi\rangle}{m_{\pi}\left\langle 0\left|A_{4}\right| \pi\right\rangle}$ where $P$ and $A_{4}$ are the pseudoscalar quark bilinear operator and the temporal component of the axial-vector operator, respectively. Pion two-point correlators are calculated to obtain the corresponding MEs. Figure 11 shows the ratio of $Z_{A}$ as a function of Euclidean time of the pion correlators at the unitary point in the left panel and the chiral extrapolation in the right panel. The final value we get is $Z_{A}(32 \mathrm{ID})=1.141(1)$.

The results of $Z_{A}^{\mathrm{D}, \mathrm{RI}}$ and $Z_{A}^{\mathrm{D}, \overline{\mathrm{MS}}}$ are plotted in Figs. 12-14 for the three lattices we use, respectively. In each figure, the

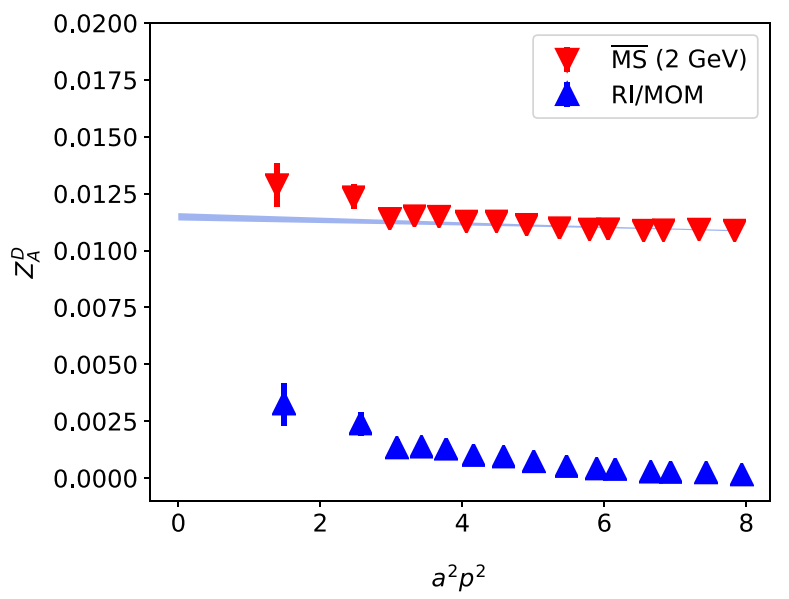

left panel shows the $a^{2} p^{2}$ dependence of $Z_{A}^{\mathrm{D}, \mathrm{RI}}$ and also the remaining $a^{2} p^{2}$ effects of $Z_{A}^{\mathrm{D}, \overline{\mathrm{MS}}}$ after we match to the $\overline{\mathrm{MS}}$ scheme at $\mu=2 \mathrm{GeV}$ from the RI-MOM results at $p^{2}$ scale. All the $Z_{A}^{\mathrm{D}, \mathrm{RI}}$ in the figures are already extrapolated to the chiral limit by a linear fit to $m_{q} a$. The right panel of these figures shows this linear extrapolation for three typical values of $a^{2} p^{2}$. The blue bands show the linear fit results of either the $a^{2} p^{2}$ dependence or the $m_{a} a$ dependence; all the $\chi^{2} /$ d.o.f. of the fits are less than 1 . For the fitting of the $a^{2} p^{2}$ dependence, small $a^{2} p^{2}$ values are excluded since the renormalization scale of these points is not large enough such that the two-loop matching factor can have a large truncation error. The final values we achieve at $2 \mathrm{GeV}$ are $Z_{A}^{\mathrm{D}, \overline{\mathrm{MS}}}(32 \mathrm{I})=0.01148(16), Z_{A}^{\mathrm{D}, \overline{\mathrm{MS}}}(24 \mathrm{I})=0.01517(88)$, and $Z_{A}^{\mathrm{D}, \overline{\mathrm{MS}}}(32 \mathrm{ID})=0.01709(45)$, respectively.

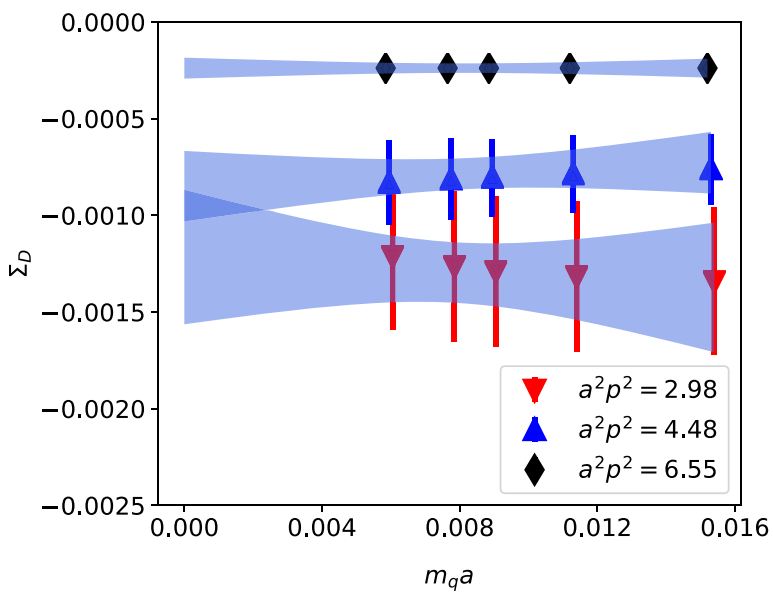

FIG. 12. Renormalization calculation on the 32I lattice. The left panel shows the $a^{2} p^{2}$ dependence of $Z_{A}^{\mathrm{D}, \mathrm{RI}}$ and also the remaining $a^{2} p^{2}$ effects of $Z_{A}^{\mathrm{D}, \overline{\mathrm{MS}}}$ after matching to the $\overline{\mathrm{MS}}$ scheme at $\mu=2 \mathrm{GeV}$. The blue band of the left plot shows the linear extrapolation of $Z_{A}^{\mathrm{D}, \mathrm{MS}}$; the first two points are not included. The right panel shows the $m_{q} a$ dependence and the linear chiral extrapolation of $\Sigma_{D}$ at three typical $a^{2} p^{2}$ values. 

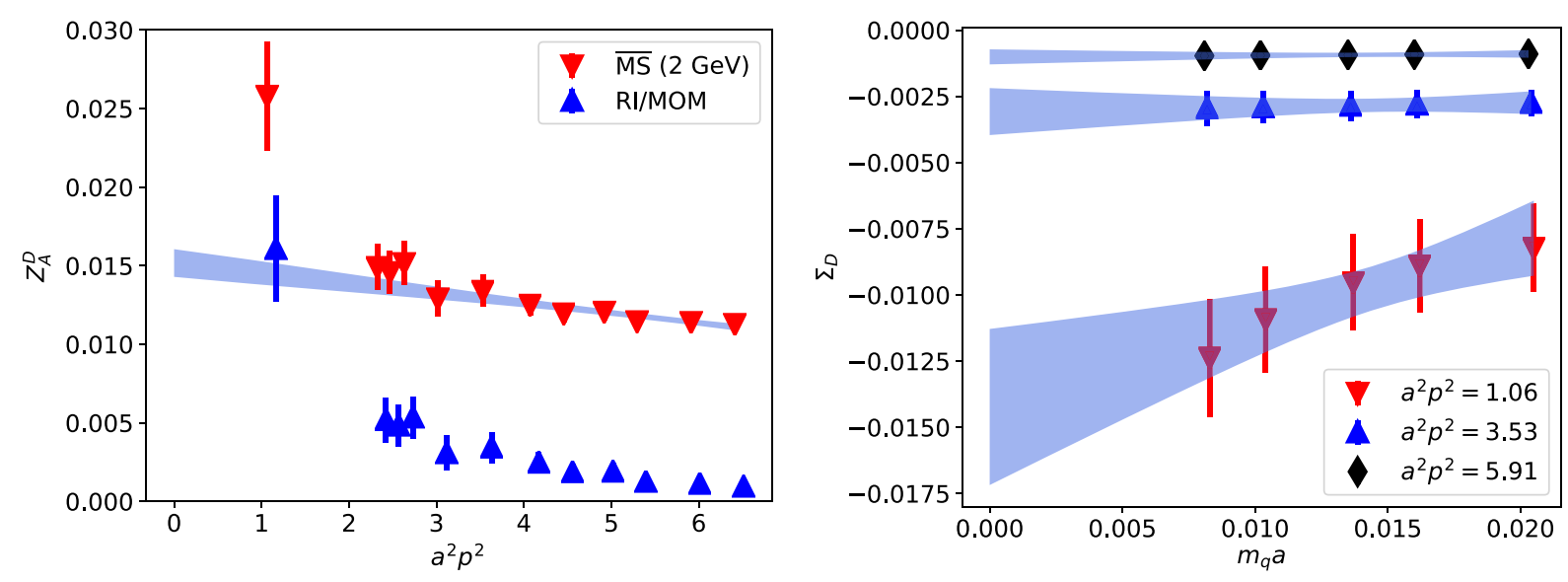

FIG. 13. The same as Fig. 12 but for the 24I lattice.
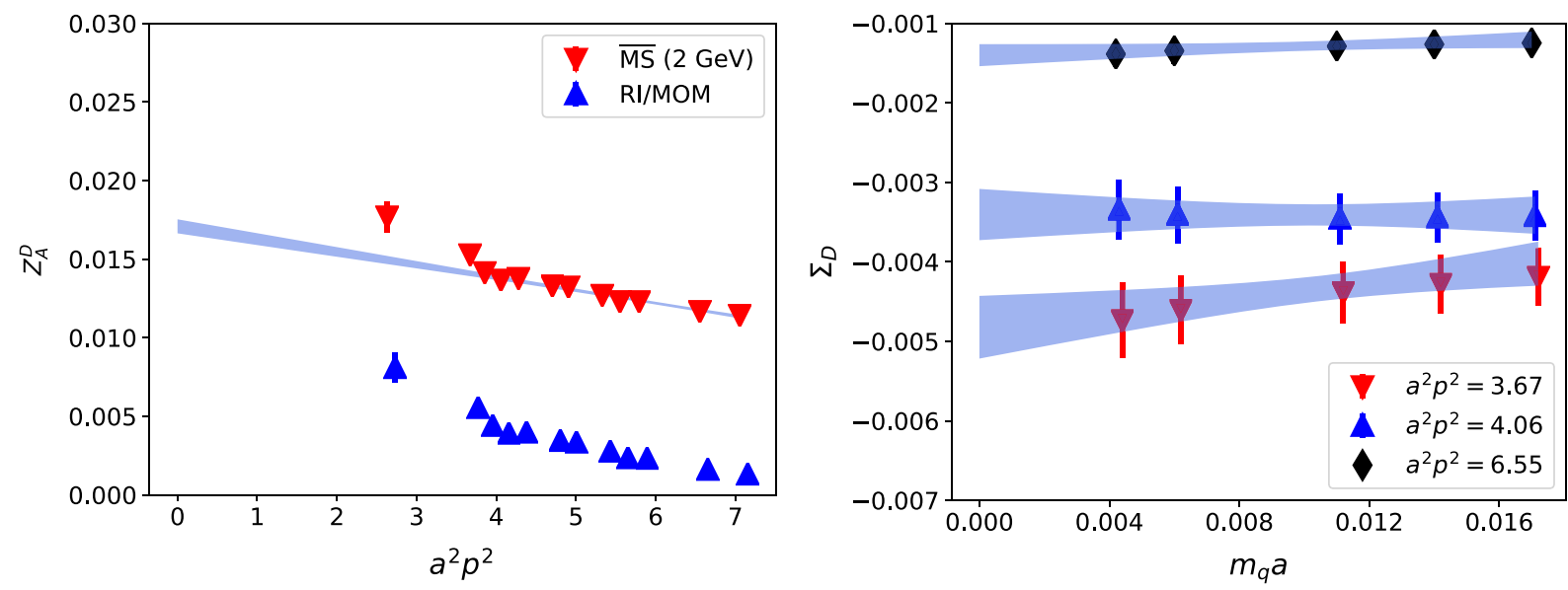

FIG. 14. The same as Fig. 12 but for the 32ID lattice.

The CDER technique is also used in the calculation of all the disconnected parts of the vertex functions. Since the overall correction of this part is small, we do not need very precise results, and so no aggressive cutoff is applied. In practice, the cutoffs are chosen to be $22 a, 38 a$, and $15 a$ for the 24I, 32I, and 32ID lattices, respectively. The improvement of the signal-to-noise ratio is $\sim 50 \%$ or less. The criterion of choosing the cutoff is based on the $\chi^{2}$ of the linear fit with respect to $a^{2} p^{2}$, which is described in detail in Ref. [42].

\section{GLOBAL FITTING AND RESULTS}

Having the bare MEs and the renormalization constants we obtained in the previous sections, we can now carry out the global fitting to push our results to the physical pion point, the continuum limit, and the infinite volume limit. The functional form used is

$$
\begin{aligned}
g_{A}= & c_{0}+c_{1}^{\mathrm{I}} / c_{1}^{\mathrm{ID}} a^{2}+c_{2}\left(m_{\pi, v}^{2}-m_{\pi, p}^{2}\right) \\
& +c_{3}\left(m_{\pi, s}^{2}-m_{\pi, p}^{2}\right)+c_{4} e^{-m_{\pi, v} L},
\end{aligned}
$$

where $m_{\pi, v}^{2}$ means the valence pion mass, $m_{\pi, s}^{2}$ means the sea pion mass, and $L$ is the size of the lattice. We have two $m_{\pi}^{2}$ terms in the fitting since we are using partially quenched valence quark masses. We use two coefficients $c_{1}^{\mathrm{I}} / c_{1}^{\mathrm{ID}}$ for the lattice spacing dependence term which reflects the fact that the ensembles we are using are generated with two slightly different gauge actions (Iwasaki for 24I and 32I and Iwasaki plus DSDR for 32ID). We use the form as $\left(m_{\pi, v}^{2}-m_{\pi, p}^{2}\right)$ where $m_{\pi, p}^{2}$ is the physical pion mass in order to let $c_{0}=g_{A}^{\text {phy }}$ be the value in the physical limit. However, not all the coefficients in the fitting function have statistical significance during the fit, meaning that the lattice data have no constraint on the corresponding term, or in other words, the effect of the corresponding term is weak enough to be ignored with the current statistical uncertainty. To be specific, the coefficient $c_{3}$ has no statistical significance in all the cases, so we exclude this term in all fittings to avoid overfitting. The difference between the results with and without the $c_{3}$ term is included in the systematic uncertainties. The other four terms (although not all of them have signals) are all kept in 
TABLE V. The results of the coefficients and the corresponding $\chi^{2} /$ d.o.f. in the global fitting for the DI case. Results for both the light quark and the strange quark are listed.

\begin{tabular}{ccccrc}
\hline \hline & $c_{0}$ & $c_{1}^{\mathrm{I}} / c_{1}^{\mathrm{ID}}$ & $c_{2}$ & $c_{4}$ & $\chi^{2} /$ d.o.f. \\
\hline$l$ & $-0.070(12)$ & $0.64(79) / 0.97(44)$ & $0.131(51)$ & $0.11(31)$ & 0.39 \\
$s$ & $-0.035(06)$ & $0.20(29) / 0.35(21)$ & $0.024(29)$ & $-0.06(23)$ & 0.41 \\
\hline \hline
\end{tabular}

TABLE VI. The results of the coefficients and the corresponding $\chi^{2} /$ d.o.f. in the global fitting for the CI case for both $d$ and $u$ quarks.

\begin{tabular}{lccccc}
\hline \hline & $c_{0}$ & $c_{1}^{\mathrm{I}} / c_{1}^{\mathrm{ID}}$ & $c_{2}$ & $c_{4}$ & $\chi^{2} /$ d.o.f. \\
\hline$d$ & $-0.337(10)$ & $-0.087(90) /-0.006(90)$ & $0.25(10)$ & $-0.17(48)$ & 0.15 \\
$u$ & $0.917(13)$ & $0.060(60) / 0.061(60)$ & $-0.01(11)$ & $-0.56(51)$ & 0.04 \\
\hline \hline
\end{tabular}

the fitting such that our final predictions are in the physical limit. Since we use the improved axial-vector current and the finite lattice spacing effects are very weak, additional prior values for the coefficients of the $a^{2}$ terms are used to guarantee stable results. We use the fitting results without separating the lattice spacing dependence into two groups as the central value of the prior and the widths are set to be $100 \%$ of the central value. The final results of the
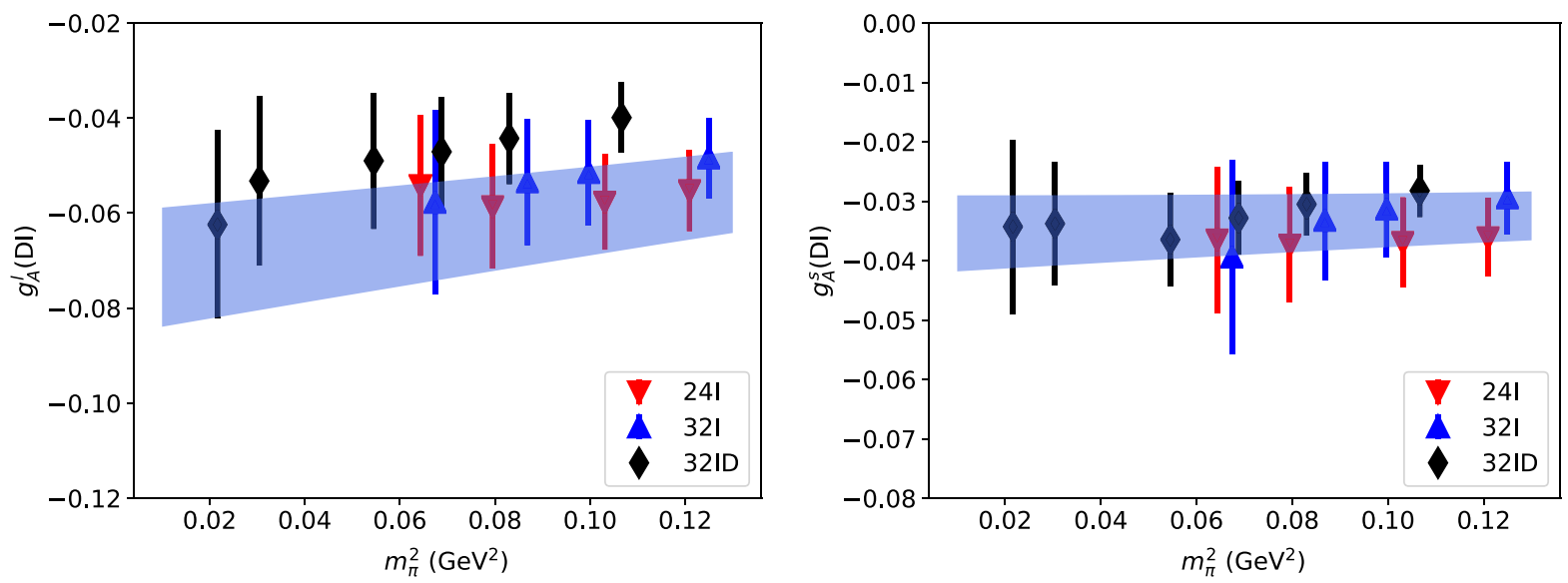

FIG. 15. The global fit of the DI case for both light and strange quarks.
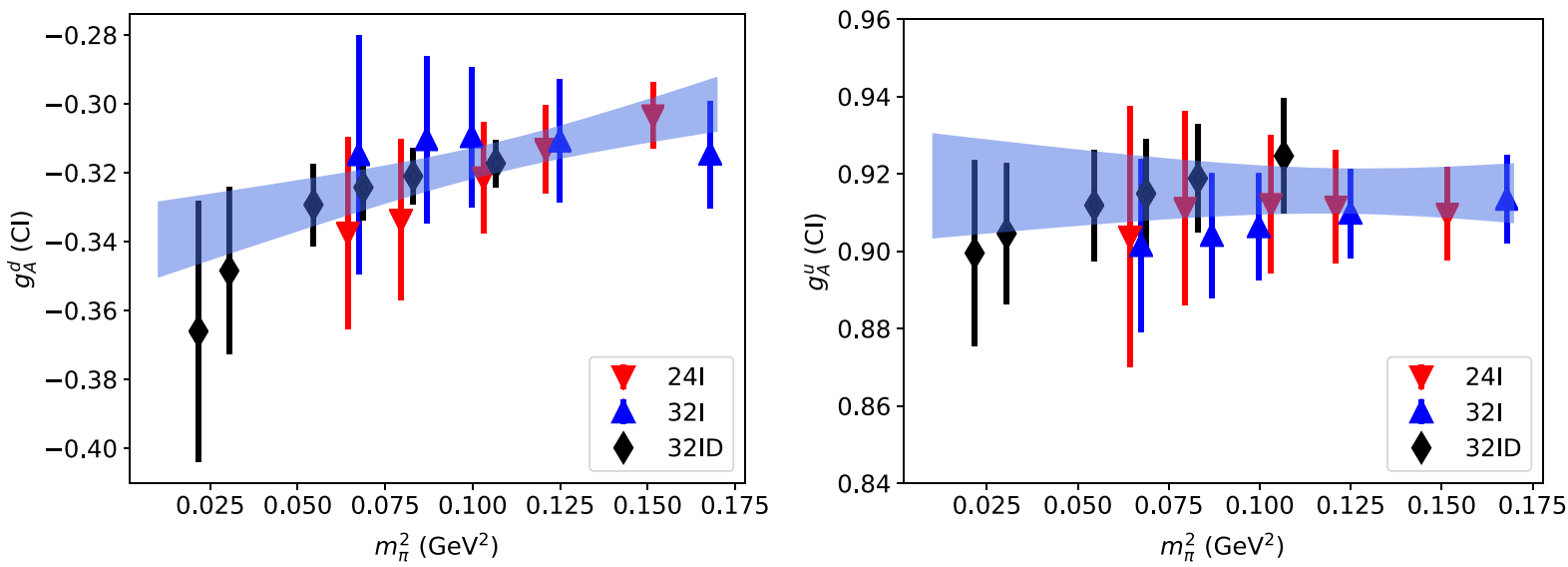

FIG. 16. The global fit of the CI case for both $d$ and $u$ quarks. 
TABLE VII. The final results of quark spin matched to the MTS scheme at $2 \mathrm{GeV}$. The errors of $g_{A}^{3}$ and $\Delta \Sigma$ are combined from the errors of $\Delta u, \Delta d$, and $\Delta s$ in quadrature. Results from two recent lattice calculations by Green et al. [34] and the Cyprus group [6] and three experimental results from de Florian et al. [3], the NNPDF Collaboration [4], and the COMPASS Collaboration [5] are also listed for comparison.

\begin{tabular}{|c|c|c|c|c|c|c|}
\hline & $\Delta u$ & $\Delta d$ & $\Delta s$ & $g_{A}^{3}$ & $g_{A}^{8}$ & $\Delta \Sigma$ \\
\hline de Florian et al. $\left(Q^{2}=10 \mathrm{GeV}^{2}\right)$ & $0.793_{-0.034}^{+0.028}$ & $-0.416_{-0025}^{+0.035}$ & $-0.012_{-0062}^{+0.056}$ & & & $0.366_{-0.062}^{+0.042}$ \\
\hline NNPDFpol1.1 $\left(Q^{2}=10 \mathrm{GeV}^{2}\right)$ & $0.76(4)$ & $-0.41(4)$ & $-0.10(8)$ & & & $0.25(10)$ \\
\hline COMPASS $\left(Q^{2}=3 \mathrm{GeV}^{2}\right)$ & {$[0.82,0.85]$} & {$[-0.45,-0.42]$} & {$[-0.11,-0.08]$} & $1.22(5)(10)$ & & {$[0.26,0.36]$} \\
\hline Green et al. & $0.863(7)(14)$ & $-0.345(6)(9)$ & $-0.0240(21)(11)$ & $1.206(7)(21)$ & $0.565(11)(13)$ & $0.494(11)(15)$ \\
\hline Alexandrou et al. & $0.830(26)(4)$ & $-0.386(16)(6)$ & $-0.042(10)(2)$ & $1.216(31)(7)$ & $0.526(39)(10)$ & $0.402(34)(10)$ \\
\hline$\chi \mathrm{QCD}$ (this work) & $0.847(18)(32)$ & $-0.407(16)(18)$ & $-0.035(6)(7)$ & $1.254(16)(30)$ & $0.510(27)(39)$ & $0.405(25)(37)$ \\
\hline
\end{tabular}

coefficients of the DI case are listed in Table V; corresponding results are also collected in Table VI for the CI case.

The final results of global fitting are shown in Figs. 15 and 16, respectively, for DI and CI. The blue bands show the fitting results with only valence pion mass dependence. Table VII lists the $\overline{\mathrm{MS}}$ numbers at $2 \mathrm{GeV}$ with both statistical and systematic errors. The systematic errors are estimated by combining the systematic uncertainties coming from the CDER technique, the fitting windows and function forms, the extrapolations, and the excited-states contamination. To be specific, for the CI case, the systematic error coming from varying fitting windows and function forms is estimated to be $3 \%$. For the DI case, the total systematic error is estimated to be $20 \%$. The final errors of $g_{A}^{3}$ and $\Delta \Sigma$ are combined from the errors of $\Delta u$, $\Delta d$, and $\Delta s$ in quadrature. Two sets of results from recent lattice calculations and three sets of results from recent global fittings of experiments are also listed in Table VII for comparison. The results from de Florian et al. [3] and NNPDFpol1.1 [4] are at $Q^{2}=10 \mathrm{GeV}^{2}$, and the integration range over the momentum fraction is from $10^{-3}$ to 1 . The COMPASS results [5] are at scale $Q^{2}=3 \mathrm{GeV}^{2}$. All the lattice results are calculated in the $\overline{\mathrm{MS}}$ scheme at $\mu=2 \mathrm{GeV}$. Since the evolution of $\Delta \Sigma$ involves a two-loop anomalous dimension [Eq. (39)], it does not vary much from $\mu=2 \mathrm{GeV}$ to $\mu=3 \mathrm{GeV}$. The calculation by Alexandrou et al. [6] is carried out on one ensemble at the physical point with two-flavor clover-improved twisted mass fermions, and the calculation by Green et al. [34] is on one ensemble with $2+1$-flavor clover fermions at $m_{\pi}=317 \mathrm{MeV}$. The total quark spin contribution of our present calculation is $\Sigma=0.405(25)(37)$, which agrees with that of Alexandrou et al. $(\sim 0.402)$ and is consistent with the experimental results. The isovector $g_{A}^{3}=$ $1.254(16)(30)$ agrees with the PDG value of 1.2723(23) within one sigma. It has a combined statistical and systematic error of $\sim 3 \%$. This is consistent with the recent percent-level lattice calculation [43] at 1.271(13), but in contrast with the other two lattice calculations in Table VII, which are lower than the experimental value. There is another lattice calculation from the JLQCD Collaboration [44] that uses dynamical overlap fermions at a single lattice spacing with four pion masses in the range $290-540 \mathrm{MeV}$. Their results $g_{A}=1.123(28)(95), \Delta s=-0.046(26)(9)$, and $\Delta \Sigma=0.398(86)(99)$ are all consistent with ours.

\section{SUMMARY}

In this work, we calculate the quark spin using overlap valences on three RBC/UKQCD domain-wall ensembles 24I, 32I, and 32ID. The pion mass of 32ID is around $171 \mathrm{MeV}$, which is close to the physical point. The anomalous Ward identity is checked carefully, and we find that the identity holds very well in our calculation with normalized axial-vector current if the divergence of the axial-vector current is inserted as an operator between nucleon states. This is an important check indicating that the lattice artifacts are under control. For the disconnectedinsertion part, the CDER technique is used for the 32ID lattice when constructing three-point functions and the statistical error can be reduced by $10 \%-40 \%$. The DI contributions to the light and strange quarks $\Delta l(\mathrm{DI})$ and $\Delta s(\mathrm{DI})$ are determined to be $-0.070(12)(15)$ and $-0.035(6)(7)$, respectively. For the connected-insertion part, we use the improved axial-vector current aiming to reduce the finite lattice spacing effects. The results of the CI contribution to $u$ and $d$ quarks $\Delta u(\mathrm{CI})$ and $\Delta d(\mathrm{CI})$ are $0.917(13)(28)$ and $-0.337(10)(10)$, respectively. As we mentioned in Sec. VII, they are scale independent due to the chiral Ward identity and can be compared to other lattice calculations. They can be extracted from deep inelastic scattering, provided the connected-sea and disconnected-sea partons are separated in the global fit [41]. Nonperturbative renormalization is carried out so the reported results are all in the $\overline{\mathrm{MS}}$ scheme at $2 \mathrm{GeV}$ scale. The numerical results are collected in Table VII; the total intrinsic quark spin contribution is $\Delta \Sigma=0.405(25)$ (37), which is consistent with the recent global fitting results of experimental data [3-5]. The isovector $g_{A}^{3}=1.254(16)(30)$ with $\sim 3 \%$ combined statistical and systematic error is within one sigma of that of the experimental value at $1.2723(23)$. 
When checking the axial Ward identity, we find that the effects of the excited states are crucial to understand the violation of the extended Goldberger-Treiman relation and even a two-term fit cannot always extract the MEs unbiasedly, so our estimations of the systematic uncertainties are relatively large. Our results can be further improved by carrying out the same calculation at the physical point directly and by using larger source-sink separations to reduce the excited-states contamination.

\section{ACKNOWLEDGMENTS}

We thank the RBC and UKQCD Collaborations for providing their DWF gauge configurations. This work is supported in part by the U.S. DOE Grant No. DESC0013065. Y. Y. is supported by the U.S. National Science Foundation under Grant No. PHY 1653405
"CAREER: Constraining Parton Distribution Functions for New-Physics Searches." This research used resources of the Oak Ridge Leadership Computing Facility at the Oak Ridge National Laboratory, which is supported by the Office of Science of the U.S. Department of Energy under Contract No. DE-AC05-00OR22725. This work used Stampede time under the Extreme Science and Engineering Discovery Environment (XSEDE), which is supported by National Science Foundation Grant No. ACI1053575. We also thank the National Energy Research Scientific Computing Center (NERSC) for providing HPC resources that have contributed to the research results reported within this paper. We acknowledge the facilities of the USQCD Collaboration used for this research in part, which are funded by the Office of Science of the U.S. Department of Energy.
[1] J. Ashman et al. (European Muon Collaboration), Phys. Lett. B 206, 364 (1988).

[2] J. Ashman et al. (European Muon Collaboration), Nucl. Phys. B328, 1 (1989).

[3] D. de Florian, R. Sassot, M. Stratmann, and W. Vogelsang, Phys. Rev. D 80, 034030 (2009).

[4] E. R. Nocera, R. D. Ball, S. Forte, G. Ridolfi, and J. Rojo (NNPDF Collaboration), Nucl. Phys. B887, 276 (2014).

[5] C. Adolph et al. (COMPASS Collaboration), Phys. Lett. B 753, 18 (2016).

[6] C. Alexandrou, M. Constantinou, K. Hadjiyiannakou, K. Jansen, C. Kallidonis, G. Koutsou, A. Vaquero AvilésCasco, and C. Wiese, Phys. Rev. Lett. 119, 142002 (2017).

[7] K.-F. Liu, J. Liang, and Y.-B. Yang, Phys. Rev. D 97, 034507 (2018).

[8] K. Fujikawa, Phys. Rev. Lett. 42, 1195 (1979).

[9] J. Kodaira, S. Matsuda, K. Sasaki, and T. Uematsu, Nucl. Phys. B159, 99 (1979).

[10] D. Espriu and R. Tarrach, Z. Phys. C 16, 77 (1982).

[11] P. Hasenfratz, V. Laliena, and F. Niedermayer, Phys. Lett. B 427, 125 (1998).

[12] Y. Kikukawa and A. Yamada, Phys. Lett. B 448, 265 (1999).

[13] D. H. Adams, Ann. Phys. (Amsterdam) 296, 131 (2002).

[14] K. Fujikawa, Nucl. Phys. B546, 480 (1999).

[15] H. Suzuki, Prog. Theor. Phys. 102, 141 (1999).

[16] P. Hasenfratz, S. Hauswirth, T. Jorg, F. Niedermayer, and K. Holland, Nucl. Phys. B643, 280 (2002).

[17] H. Neuberger, Phys. Lett. B 417, 141 (1998).

[18] P. H. Ginsparg and K. G. Wilson, Phys. Rev. D 25, 2649 (1982).

[19] T.-W. Chiu, Phys. Rev. D 60, 034503 (1999).

[20] K.-F. Liu, Int. J. Mod. Phys. A 20, 7241 (2005).

[21] M. F. Atiyah and I. M. Singer, Ann. Math. 93, 139 (1971).
[22] A. Li et al. (xQCD Collaboration), Phys. Rev. D 82, 114501 (2010).

[23] Y. Aoki et al. (RBC Collaboration and UKQCD Collaboration), Phys. Rev. D 83, 074508 (2011).

[24] T. Blum et al. (RBC Collaboration and UKQCD Collaboration), Phys. Rev. D 93, 074505 (2016).

[25] Y.-B. Yang, A. Alexandru, T. Draper, M. Gong, and K.-F. Liu, Phys. Rev. D 93, 034503 (2016).

[26] J. Liang, Y.-B. Yang, K.-F. Liu, A. Alexandru, T. Draper, and R. S. Sufian, Phys. Rev. D 96, 034519 (2017).

[27] M. Gong et al. ( $\chi$ QCD Collaboration), Phys. Rev. D 88, 014503 (2013).

[28] M. Gong, Y.-B. Yang, J. Liang, A. Alexandru, T. Draper, and K.-F. Liu ( $\chi$ QCD Collaboration), Phys. Rev. D 95, 114509 (2017).

[29] Y.-B. Yang, A. Alexandru, T. Draper, J. Liang, and K.-F. Liu ( $\chi$ QCD Collaboration), Phys. Rev. D 94, 054503 (2016).

[30] Y.-B. Yang et al., Phys. Rev. D 92, 034517 (2015).

[31] Z. Liu, Y. Chen, S.-J. Dong, M. Glatzmaier, M. Gong, A. Li, K.-F. Liu, Y.-B. Yang, and J.-B. Zhang ( $\chi$ QCD Collaboration), Phys. Rev. D 90, 034505 (2014).

[32] R. Gupta, Y.-C. Jang, H.-W. Lin, B. Yoon, and T. Bhattacharya, Phys. Rev. D 96, 114503 (2017).

[33] G. Martinelli, C. Pittori, C. T. Sachrajda, M. Testa, and A. Vladikas, Nucl. Phys. B445, 81 (1995).

[34] J. Green, N. Hasan, S. Meinel, M. Engelhardt, S. Krieg, J. Laeuchli, J. Negele, K. Orginos, A. Pochinsky, and S. Syritsyn, Phys. Rev. D 95, 114502 (2017).

[35] H. H. Patel, Comput. Phys. Commun. 197, 276 (2015).

[36] H. H. Patel, Comput. Phys. Commun. 218, 66 (2017).

[37] G. M. Prosperi, M. Raciti, and C. Simolo, Prog. Part. Nucl. Phys. 58, 387 (2007).

[38] K.-F. Liu and S.-J. Dong, Phys. Rev. Lett. 72, 1790 (1994).

[39] K.-F. Liu, Phys. Rev. D 62, 074501 (2000). 
[40] K.-F. Liu, W.-C. Chang, H.-Y. Cheng, and J.-C. Peng, Phys. Rev. Lett. 109, 252002 (2012).

[41] K.-F. Liu, Phys. Rev. D 96, 033001 (2017).

[42] Y.-B. Yang, M. Gong, J. Liang, H.-W. Lin, K.-F. Liu, D. Pefkou, and P. Shanahan, arXiv:1805.00531.
[43] C. C. Chang et al., Nature (London) 558, 91 (2018).

[44] N. Yamanaka, S. Hashimoto, T. Kaneko, and H. Ohki (JLQCD Collaboration), Phys. Rev. D 98, 054516 (2018). 